\title{
A Hydrogen Bond Between Linear Tetrapyrrole and Conserved Aspartate Causes the Far-Red Shifted Absorption of Phytochrome Photoreceptors
}

Egle Maximowitsch, Tatiana Domratcheva

Submitted date: 10/05/2020 - Posted date: 12/05/2020

Licence: CC BY-NC-ND 4.0

Citation information: Maximowitsch, Egle; Domratcheva, Tatiana (2020): A Hydrogen Bond Between Linear Tetrapyrrole and Conserved Aspartate Causes the Far-Red Shifted Absorption of Phytochrome Photoreceptors. ChemRxiv. Preprint. https://doi.org/10.26434/chemrxiv.12278780.v1

Photoswitching of phytochrome photoreceptors between red-absorbing (Pr) and far-red absorbing (Pfr) states triggers light adaptation of plants, bacteria and other organisms. Using quantum chemistry, we elucidate the color-tuning mechanism of phytochromes and identify the origin of the Pfr-state red-shifted spectrum. Spectral variations are explained by resonance interactions of the protonated linear tetrapyrrole chromophore. In particular, hydrogen bonding of pyrrole ring $D$ with the strictly conserved aspartate shifts the positive charge towards ring $\mathrm{D}$ thereby inducing the red spectral shift. Our MD simulations demonstrate that formation of the ring D-aspartate hydrogen bond depends on interactions between the chromophore binding domain (CBD) and phytochrome specific domain (PHY). Our study guides rational engineering of fluorescent phytochromes with a far-red shifted spectrum.

File list (2)

phytochromes_red_shift_Egle_Maximowitsch.pdf (1.55 MiB) view on ChemRxiv - download file 


\title{
A hydrogen bond between linear tetrapyrrole and conserved aspartate causes the far-red shifted absorption of phytochrome photoreceptors
}

\author{
Egle Maximowitsch ${ }^{[\mathrm{a}]}$ and Tatiana Domratcheva*[a]
}

\begin{abstract}
Photoswitching of phytochrome photoreceptors between red-absorbing (Pr) and far-red absorbing (Pfr) states triggers light adaptation of plants, bacteria and other organisms. Using quantum chemistry, we elucidate the color-tuning mechanism of phytochromes and identify the origin of the Pfr-state red-shifted spectrum. Spectral variations are explained by resonance interactions of the protonated linear tetrapyrrole chromophore. In particular, hydrogen bonding of pyrrole ring $D$ with the strictly conserved aspartate shifts the positive charge towards ring $D$ thereby inducing the red spectral shift. Our MD simulations demonstrate that formation of the ring D-aspartate hydrogen bond depends on interactions between the chromophore binding domain (CBD) and phytochrome specific domain (PHY). Our study guides rational engineering of fluorescent phytochromes with a far-red shifted spectrum
\end{abstract}

Phytochrome proteins function as sensors of light conditions via photoisomerization of their linear tetrapyrrole chromophore absorbing red light ${ }^{1}$. Recently, the phytochrome far-red emission has been employed in deep-tissue imaging ${ }^{2}$. To increase tissue penetration, shifting the spectral maximum of the engineered phytochromes towards higher wavelengths is necessary, but has not been achieved so far. Natural phytochromes form the far-red shifted absorption upon redlight illumination. The two photoswitchable states, the red absorbing Pr and far-red absorbing Pfr state, demonstrate a $\sim 50-\mathrm{nm}$ spectral shift that is crucial for the phytochrome biological function, as for instance, it enables plants to detect shadow conditions. The Pr and Pfr states bind the tetrapyrrole chromophore in the 5Zs10Zs15Za and 5Zs10Zs15Ea configuration, respectively ${ }^{3-4}$ (Figure 1 ). In spite of attempts by computational studies to link the spectral shift to the chromophore isomerization $^{5-8}$, the electronic origin of the red
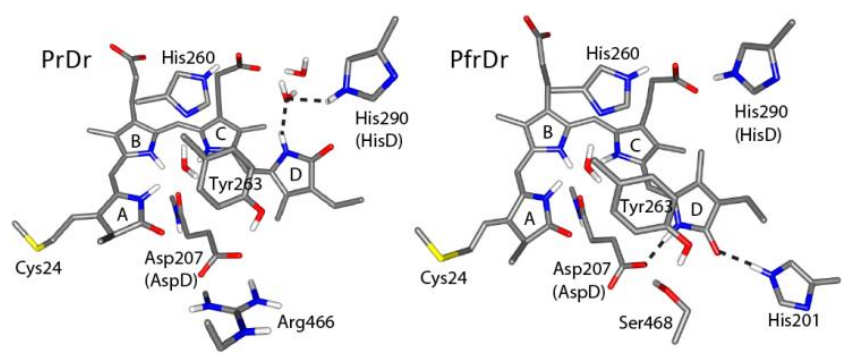

Figure 1 The linear tetrapyrrole chromophore in the Pr-and Pfr-state of DrBphP.

\footnotetext{
a. Department of Biomolecular Mechanisms, Max Planck Institute for Medical Research, Jahnstrasse 29, 69120 Heidelberg, Germany.

E-mail: Tatjana.Domratcheva@mpimf-heidelberg.mpg.de

Supplementary Information (SI) and complete reference 17 is available.
}

Pfr spectral shift remains unrevealed. Obviously, the interactions of the tetrapyrrole chromophore with the protein that differ in the $\mathrm{Pr}$ and $\operatorname{Pfr}$ states $^{9-10}$ (Figure 1) underlie spectral tuning. There are several strictly conserved polar residues surrounding the tetrapyrrole chromophore covalently bound to a cysteine residue in the protein binding pocket ${ }^{9-13}$. Here we elucidate the mechanism by which polar interactions tune the tetrapyrrole red-light absorption.

We prepared molecular cluster models representing the active sites of bacterial and plant phytochromes in the $\mathrm{Pr}$ and $\mathrm{Pfr}$ states. Plant phytochromes contain a phytochromobilin chromophore whereas bacterial bind biliverdin ${ }^{1}$. Biliverdin - a product of heme degradation - is present at abundance in tissues; whereas phytochromobilin is specifically produced by a double-bond reduction of biliverdin ring $A$ in plants. This covalent modification of the cofactor shifts the tetrapyrrole red-absorption maximum to lower wavelengths. We chose plant and bacterial phytochrome models to compare the red Pr-Pfr shift in different phytochromes and also to consider the origin of the blue shift in plant phytochromes with respect to bacterial ones.

We selected phytochromes from bacteria $D$. radiodurans and $P$. aeruginosa (DrBphP and $\mathrm{PaBphP}$, respectively), for which the $\mathrm{Pr}$ - and/or Pfr-state structures have been reported ${ }^{9-12}$, and phytochrome B from the plant $A$. thaliana (AtPhyB), for which
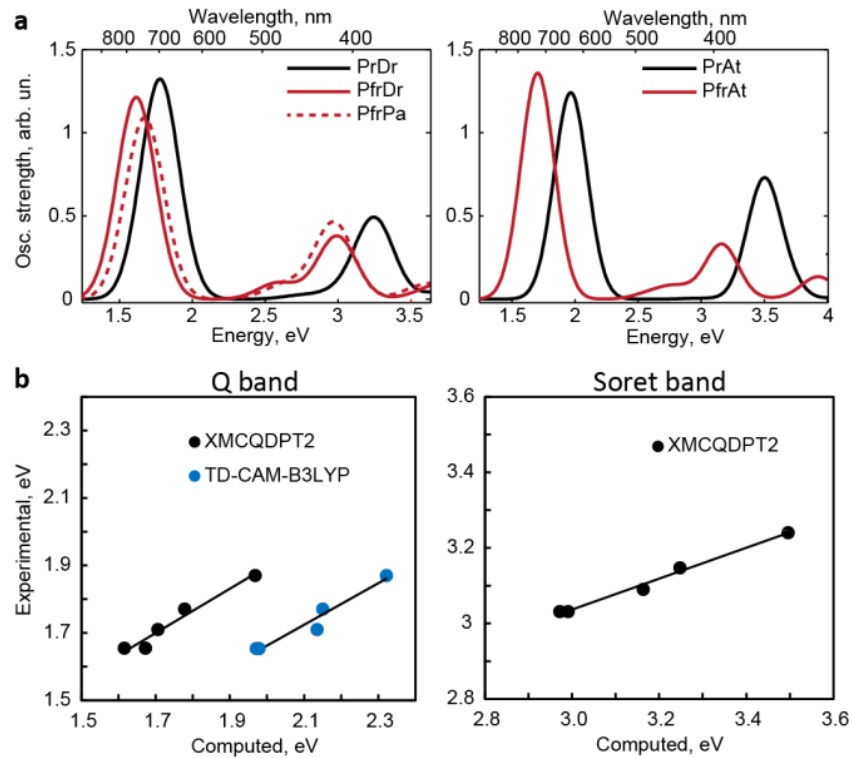

Figure 2 Computed phytochrome excitation spectra. (a) Spectral shapes obtained by Gaussian broadening ( $0.3 \mathrm{eV}$ FWHM) of the XMCQDPT2 excitation energies and oscillator strength. (b) Correlation between the computed excitation energies and experimental $Q$ - and Soret-band maxima. 
Table 1. The So-S1 energies [eV] and oscillator strength (indicated in brackets) computed with the XMCQDPT2 method in comparison to the energies of the experimental Q-band maximum.

\begin{tabular}{cccccc}
\hline Model & Pr DrBphP & Pfr DrBphP & Pfr PaBphP & Pr AtPhyB & Pfr AtPhyB \\
\hline Experimental & $1.77^{19}$ & $1.65^{19}$ & $1.65^{20}$ & $1.87^{13}$ & $1.71^{13}$ \\
\hline Cluster WT & $1.78(1.323)$ & $1.62(1.214)$ & $1.67(1.091)$ & $1.97(1.243)$ & $1.71(1.360)$ \\
\hline Chrom-extracted & $1.85(1.368)$ & $1.73(1.237)$ & $1.73(1.119)$ & $2.02(1.312)$ & $1.87(1.367)$ \\
\hline Cluster w/o AspD & $1.77(1.224)$ & $1.70(1.188)$ & $1.75(1.028)$ & $1.93(1.254)$ & $1.92(1.297)$ \\
\hline Chrom-opt & $1.68(1.024)$ & $1.64(1.006)$ & $1.66(0.908)$ & $1.74(1.079)$ & $1.68(1.012)$ \\
\hline
\end{tabular}

only the Pr-state crystal structure was reported ${ }^{13}$. To prepare the Pfr model of AtPhyB, we assumed the ring D rotation in the plant homolog to be similar to that in the bacterial one (Figure 1). The PBEO-D3 method $^{14-15}$ was chosen for geometry optimization. The excitation energies and molecular properties were computed with several methods as specified below. The calculations were performed using the Firefly ${ }^{16}$ and Gaussian ${ }^{17}$ software. For further details see the Supplementary Information (SI).

Our calculations of the active-site cluster models (indicated as "cluster WT" in Table 1) using the XMCQDPT2 method ${ }^{18}$ reproduced the shape of the experimental spectrum consisting of the $\mathrm{Q}$ and Soret bands ${ }^{13,19-20}$ (Figure 2a). The Q-band maximum $\left(630-770 \mathrm{~nm}\right.$ ) is defined by the $\mathrm{S}_{0}-\mathrm{S}_{1}$ energy (Table 1). The Pfr-state energy decreases with respect to that of the Pr-state by $0.16 \mathrm{eV}$ for bacterial DrBphP and $0.26 \mathrm{eV}$ for plant AtPhyB models. Also, the energies of the plant models are higher than those of the bacterial models. The correlation plot demonstrates excellent agreement of the computed and experimental spectral shifts (Figure $2 b$ ). The experimental Qband shifts were also reproduced by the TD-CAM-B3LYP ${ }^{21}$ calculations (Figure $2 \mathrm{~b}$ ) although the computed energies are systematically higher than the experimental ones. The overestimation of the energies with a TD-DFT method is consistent with previous reports ${ }^{22-23}$. The Soret-band maximum (350-420 $\mathrm{nm}$ ) cannot be reliably predicted by the TD-CAM-B3LYP calculations, indicating contributions from double- and higher-order excitations. In contrast, the Q-band shifts could be attributed to energy change of the singleelectronic excitations, as demonstrated by correlation of the $\mathrm{S}_{0}-\mathrm{S}_{1}$ energy with the HOMO-LUMO energy gap (Figure $\mathrm{S} 1$ ). The excitation energies obtained with the CIS method for bacterial models (Table S1), although largely overestimated, also predict the correct Pr-Pfr shift.

To study the spectral shift origin, we first computed excitation energies of the chromophores without the propionate groups extracted from the cluster models without changing their geometries (indicated as "Chrom-extracted" in Table 1). These calculations yielded a significant Pr-Pfr difference of $0.12 \mathrm{eV}$ (DrBphP models) and $0.15 \mathrm{eV}$ (AtPhyB models), suggesting that changes in the chromophore electronic structure underlie the spectral difference. Further, we studied the impact of interactions between the chromophore and highly conserved aspartate (AspD in Figure 1). The geometry optimization and excitation-energy calculations were performed for models which did not contain the AspD carboxylic group (indicated as "Cluster w/o AspD" in Table 1). We found a substantially reduced Pr-Pfr red shift of $0.07 \mathrm{eV}$ (DrBphP models w/o AspD) and $0.01 \mathrm{eV}$ (AtPhyB models w/o AspD). The ring $D-A s p D$ interactions decrease the Q-band energy in the Pfr state, whereas they hardly affect or slightly increase the energy in the Pr state. In addition, we demonstrated that the hydrogen bond between the semi-conserved histidine residue (HisD in Figure 1) and ring $D$ does not contribute to the lowering of the $\mathrm{S}_{0}-\mathrm{S}_{1}$ energy of the Pr state. In the Pr DrBphP model, change of protonation or removing HisD practically does not change the energy (Table S1). In the Pr AtPhyB model, protonated HisD increases the energy by $0.09 \mathrm{eV}$, whereas the absence of HisD decreases the energy by $0.02 \mathrm{eV}$ (Table S1). Finally, we again considered the biliverdin and phytochromobilin chromophores without propionates but this time after their geometries were optimized starting from the crystal structure coordinates (indicated as "Chrom-opt" in Table 1). The 15Ea and 15Za isomers were different in their $\mathrm{S}_{0}-\mathrm{S}_{1}$ energy only by $0.04 \mathrm{eV}$ and $0.06 \mathrm{eV}$ in the case of biliverdin and phytochromobilin, respectively.

Retention of the spectral shift in the extracted chromophores and its disappearance in the optimized chromophores motivated us to compare the geometry of the tetrapyrrole moiety in the models. After geometry optimization, the dihedral angles of the methine bridges are very close to those in the parent crystal structures (Table S2) and the pyrrole rings are essentially planar (Figure S2). However, we found a characteristic bond-length alternation pattern when comparing the tetrapyrrole of the Pr-and Pfr models (Figure 3a and Table S3). The largest in amplitude change is displayed by the CD methine bridge, specifically the C14-C15 distance shortens whereas the $\mathrm{C} 15-\mathrm{C} 16$ distance elongates in the $\mathrm{Pfr}$ as compared to the $\operatorname{Pr}$ models. This noticeable difference persisted neither upon removing AspD (Figure 3a) nor in the optimized chromophores (Figure S3). Hence, interactions of the ring $D$ in the 15Ea configuration with AspD induce a significant geometry change at the $C D$ methine bridge. The geometry change indicates an electronic structure change manifested as spectral shift. 

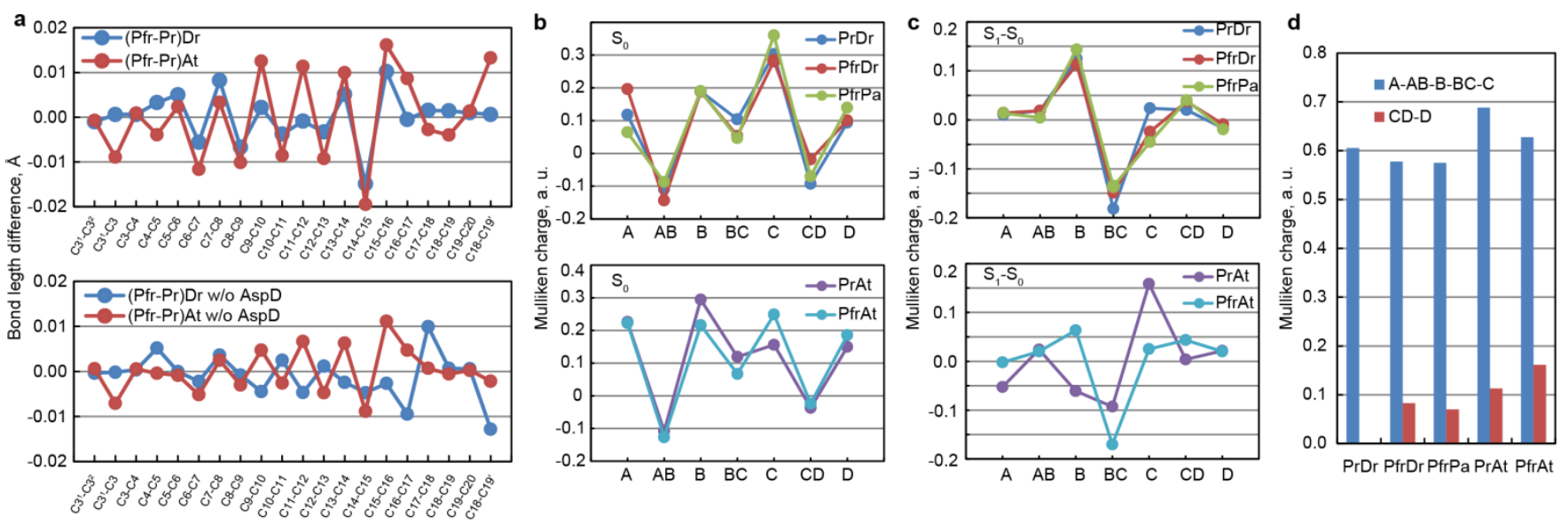

Figure 3 Changes in geometry and charge distribution underlying spectral shifts. (a) Comparison of the bond lengths in the Pr and Pfr models. (b) Distribution of Mulliken charges on the pyrrole rings (A-D) and methine bridges ( $A B, B C$, and $C D$ ) in the SO state; (c) Redistribution of Mulliken charges upon excitation (S1-state minus SO-state). (d) Net charge on fragments A-AB-B-BC-C and BC-D in the SO state. Atom labelling in (a) is presented in Scheme 1. In (b)-(d), the charges were obtained from the XMCQDPT2 calculations.

Four resonance structures (1-4) describe delocalization of the positive charge in a protonated linear tetrapyrrole (Scheme 1). Varying contributions of 1-4 explain the bond-length variation (Figure 3a), delocalization of the positive charge (Figure $3 \mathrm{~b}$ ) and charge transfer character of the $\mathrm{S}_{0}-\mathrm{S}_{1}$ transition (Figure 3c). All these properties are presented in the Tables S1, S3-S5. The charge distribution analysis demonstrates substantial contributions of all four resonance structures. Consistently, the change of the $S_{0}$ and $S_{1}$ dipole moments (Table S1) shows only a small charge transfer character. According to the net charges (Figure $\mathbf{3 b}$ ), the contribution of $\mathbf{2}$ is larger in Pr AtPhyB than in the other models. In the Pfr models as compared to the Pr models, the shift of the positive charge from rings A-B-C to ring $D$ (Figure $3 d$ ) suggests an increased contribution of 4 . This is also confirmed by the geometry change of the CD methine bridge described above (Figure $3 a$ ) and by the increase of ring $\mathrm{C}$ electron-acceptor properties (Figure 3c). Notably, the contribution of $\mathbf{4}$ in the $\mathrm{Pfr}$ state clearly decreases in the absence of the AspD side chain in both bacterial and plant models (Table S5). Thus, we assign the formation of the Pfr spectrum to interactions of ring $D$ with the AspD counterion

Scheme 1. Resonance structures of a protonated linear tetrapyrrole.
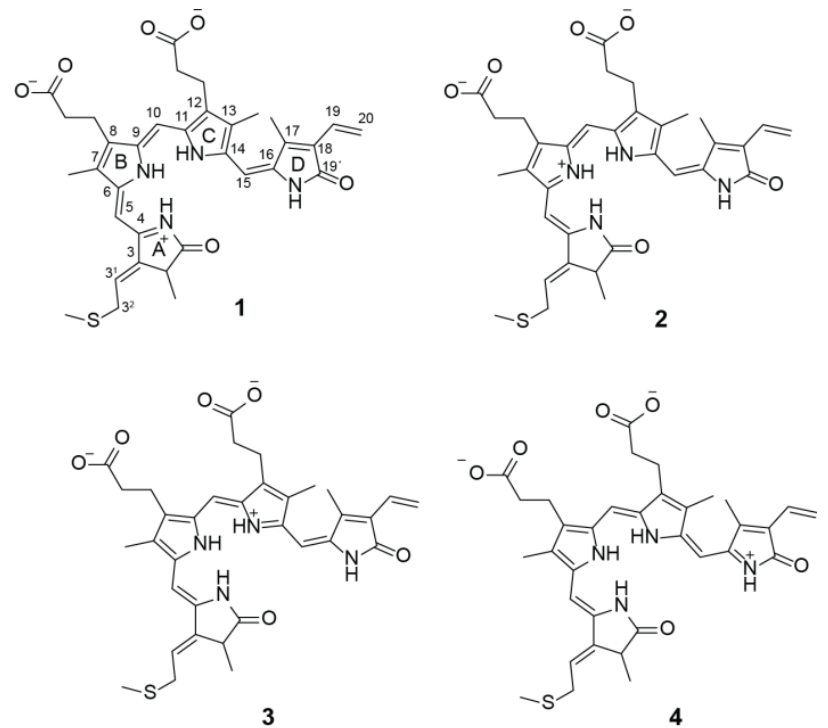

shifting the positive charge from rings $B$ (phytochromobilin) or C (biliverdin) towards ring D.

In line with our assignment, experimental evidence indicates that the highly conserved AspD residue is crucial for phytochrome photoresponse ${ }^{10,19,24-25}$. Mutations of AspD do not affect the Pr spectrum but abolish formation of the Pfr spectrum $^{19}$. Furthermore, AspD might facilitate photoconversion of the 15Za-tetrapyrrole ${ }^{24-25}$; also it might be involved in stabilization of the 15Ea-tetrapyrrole ${ }^{10}$. Yet, the phytochrome constructs that carry AspD but are truncated to the chromophore binding domain (CBD) also do not form the Pfr-spectrum ${ }^{26}$. In fact, the Pfr-spectrum was observed only for the proteins comprising both the CBD and the phytochrome specific (PHY) domain (Figure 4a). These experimental observations complemented by our assignment of the Pfr spectral shift indicate that the ring D-AspD hydrogen bond in the $\mathrm{Pfr}$ state require additional stabilization by interactions with the PHY domain.

To elucidate the role of the PHY domain, we performed MD simulations of the DrBphP and PaBphP proteins using the NAMD software ${ }^{27}$ (see the SI for computational details). In the models lacking the PHY domain, the hydrogen bonding interactions around the chromophore were clearly destabilized (Figure S4). In the truncated proteins, the solvent-exposed AspD side chain facilitates water dynamics, in line with a previous report ${ }^{28}$. We observed that AspD and a neighbouring conserved tyrosine (Tyr263 in Figure 1) constitute a gate allowing solvent water to enter the active site and exchange with the so-called pyrrole water coordinated by the tetrapyrrole moiety. To simulate hydrogen bond formation between the isomerized 15Ea ring $D$ and AspD, a series of DrBphP models was considered in which the domain structures were varied (Figure $4 \mathrm{~b}$ ). If both $\mathrm{CBD}$ and $\mathrm{PHY}$ domains retain the Pr-state structure, flipping of the ring D from 15Za to 15Ea did not result in a stable hydrogen bond between the ring $D$ and AspD. In contrast, removing Pr-PHY or replacing it by Pfr$\mathrm{PHY}$ resulted in the significantly shorter distance indicating hydrogen bond formation, because the change of the PHY 


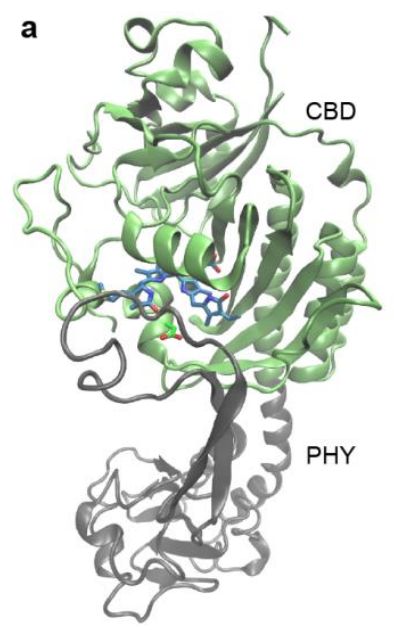

b
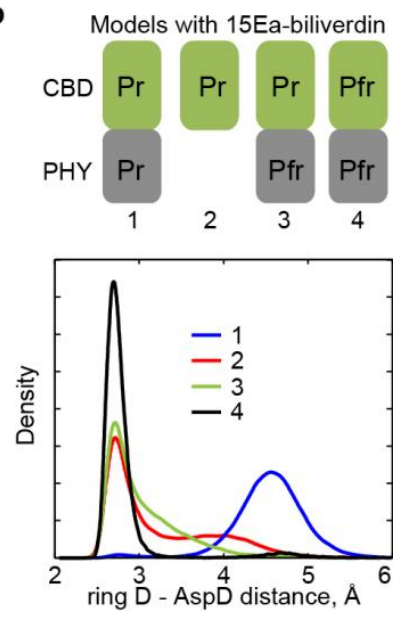

Figure 4. Stabilization of the ring $D-A s p D$ hydrogen bond by CBD-PHY interactions. (a) Structure of the phytochrome photosensory module comprising $\mathrm{CBD}$ and PHY domain. (b) The distance distribution characterizing formation of the ring D - AspD hydrogen bond after 15Za/15Ea biliverdin photoisomerization in a series of models with varied structures of $\mathrm{CBD}$ and $\mathrm{PHY}$ domain.

domain structure replaced interactions between AspD and conserved arginine in Pr-PHY with interactions between AspD and conserved serine in Pfr-PHY (Arg466 and Ser468 in Figure 1). As suggested by our MD simulations, these interactions modulate formation of the ring D-AspD hydrogen bond. Other interactions in the active site also change accompanying formation of the ring $D-A s p D$ hydrogen bond after the 15Za/15Ea biliverdin isomerization (Figures S5 and S6).

To conclude, our computational study demonstrates that resonance interactions play a role in spectral tuning of protonated linear tetrapyrroles in phytochrome photoreceptors. The red shift of the Pfr spectrum results from the shift of the positive charge towards ring $D$ by interactions with the aspartate counterion. The naturally evolved means to control these resonance interactions encompasses stabilization of the chromophore-counterion interactions by the C-terminal PHY domain. Suited for the photoreceptor function, this complex mechanism would require special engineering in order to be implemented in the red-fluorescent variants of bacterial phytochromes which typically comprise only the $\mathrm{CBD}^{2}$. The other example considered here, the blue spectral shift of the plant phytochromes as compared to the bacterial analogues is explained by the shift of the positivecharge towards pyrrole ring $\mathrm{B}$. This colour tuning, which is critically important for plants, entails a specific oxidoreductase enzyme that reduces a double bond of pyrrole ring $A^{1}$.

\section{Conflicts of interest}

There are no conflicts to declare.

\section{Notes and references}

1 N. C. Rockwell, Y. S. Su and J. C. Lagarias, Annu Rev Plant Biol, 2006, 57, 837-858.

2 K. G. Chernov, T. A. Redchuk, E. S. Omelina and V. V. Verkhushaa, Chem Rev, 2017, 117, 6423-6446.

3 J. C. Lagarias and H. Rapoport, J Am Chem Soc, 1980, 102, 4821-4828.

4 W. Rudiger, F. Thummler, E. Cmiel and S. Schneider, Proceedings of the National Academy of Sciences of the United States of America, 1983, 80, 6244-6248.

5 J. Hasegawa, M. Isshiki, K. Fujimoto and H. Nakatsuji, Chemical Physics Letters, 2005, 410, 90-93.

6 R. A. Matute, R. Contreras and L. Gonzalez, J Phys Chem Lett, 2010, 1, 796-801.

7 I. V. Polyakov, B. L. Grigorenko, V. A. Mironov and A. V. Nemukhin, Chemical Physics Letters, 2018, 710, 59-63.

8 C. Wiebeler and I. Schapiro, Molecules, 2019, 24.

9 J. R. Wagner, J. Zhang, J. S. Brunzelle, R. D. Vierstra and K. T. Forest, The Journal of biological chemistry, 2007, 282, 1229812309.

10 X. Yang, J. Kuk and K. Moffat, Proceedings of the National Academy of Sciences of the United States of America, 2008, 105, 14715-14720.

11 H. Takala, A. Bjorling, O. Berntsson, H. Lehtivuori, S. Niebling, M. Hoernke, I. Kosheleva, R. Henning, A. Menzel, J. A. Ihalainen and S. Westenhoff, Nature, 2014, 509, 245-248.

12 E. S. Burgie, J. Zhang and R. D. Vierstra, Structure, 2016, 24, 448-457.

13 E. S. Burgie, A. N. Bussell, J. M. Walker, K. Dubiel and R. D. Vierstra, Proceedings of the National Academy of Sciences of the United States of America, 2014, 111, 10179-10184.

14 C. Adamo and V. Barone, Journal of Chemical Physics, 1999, 110, 6158-6170.

15 S. Grimme, J. Antony, S. Ehrlich and H. Krieg, Journal of Chemical Physics, 2010, 132.

16 A. A. Granovsky, http://classic.chem.msu.su/gran/firefly/index.html.

17 Gaussian 09, Frisch MJ et al. Gaussian, Inc., Wallingford CT, 2016.

18 A. A. Granovsky, The Journal of chemical physics, 2011, 134, 214113.

19 J. R. Wagner, J. Zhang, D. von Stetten, M. Gunther, D. H. Murgida, M. A. Mroginski, J. M. Walker, K. T. Forest, P. Hildebrandt and R. D. Vierstra, The Journal of biological chemistry, 2008, 283, 12212-12226.

20 X. Yang, J. Kuk and K. Moffat, Proceedings of the National Academy of Sciences of the United States of America, 2009, 106, 15639-15644.

21 T. Yanai, D. P. Tew and N. C. Handy, Chemical Physics Letters, 2004, 393, 51-57.

22 O. Falklöf and B. Durbeej, Chemical Physics, 2013, 425, 1928.

23 O. Falklof and B. Durbeej, Journal of computational chemistry, 2013, 34, 1363-1374.

24 K. C. Toh, E. A. Stojkovic, I. H. M. van Stokkum, K. Moffat and J. T. M. Kennis, Physical Chemistry Chemical Physics, 2011, 13, 11985-11997.

25 H. Lehtivuori, I. Rissanen, H. Takala, J. Bamford, N. V. Tkachenko and J. A. Ihalainen, Journal of Physical Chemistry $B, 2013,117,11049-11057$.

26 E. S. Burgie, T. Wang, A. N. Bussell, J. M. Walker, H. Li and R. D. Vierstra, The Journal of biological chemistry, 2014, 289, 24573-24587.

27 J. C. Phillips, R. Braun, W. Wang, J. Gumbart, E. Tajkhorshid, E. Villa, C. Chipot, R. D. Skeel, L. Kale and K. Schulten, Journal of computational chemistry, 2005, 26, 1781-1802.

28 M. Feliks, C. Lafaye, X. Shu, A. Royant and M. Field, Biochemistry, 2016, 55, 4263-4274.

¥ We thank Chris Roome for excellent IT support. The computing time was granted by the Neumann Institute for Computing (NIC) on the supercomputer JURECA at Jülich Supercomputing Centre. 
Supplementary Information for

A hydrogen bond between tetrapyrrole and conserved aspartate causes the far-red shifted absorption of phytochrome photoreceptors

Egle Maximowitsch and Tatiana Domratcheva

Department of Biomolecular Mechanisms, Max Planck Institute for Medical Research, Jahnstrasse 29, 69120 Heidelberg, Germany

S1. Computational details (pages S2-S5)

S2. Supplementary Figures S1-S10 (pages S5-S11)

S3. Supplementary Tables S1-S9 (pages S12-S25)

S4. Complete reference 17 and supplementary references 29-41 (page S26) 


\section{S1. Computational details}

\section{S1.1 Quantum-chemistry calculations}

Computational methods and protocols. The Firefly program ${ }^{16}$ partially based on the US GAMESS $\operatorname{code}^{29}$ was used to carry out geometry optimization, XMCQDPT2 ${ }^{18}$ and CIS calculations ${ }^{30}$. The Gaussian09 program $^{17}$ was used to perform TD-DFT calculations. For visualization the ChemCraft program $^{31}$ was used.

The PBE0 density functional theory (DFT) method $^{14}$ and supplemented by the dispersion-energy correction (D3) ${ }^{15}$ was employed for geometry optimization in Cartesian coordinates using a second-order energy-gradient procedure. Optimization in Cartesian coordinates was chosen in order to obtain optimized structures as close as possible to the starting X-ray structure models without employing constraints to fix positions of terminal atoms in cluster models. At the optimized geometries, the excitation spectrum was computed. For the XMCQDPT2-CASSCF calculations, the starting molecular orbitals (MOs) were generated by the Hartree-Fock method calculations. All active-space MOs were localized on the tetrapyrrole fragment. By comparing the results obtained with different active spaces for various numbers of excited states, we chose the SA7-CASSCF(6,5) method (energy-averaging for 7 states, correlating 6 electrons in 5 MOs). State-averaging (SA) with equal weights was used in the CASSCF calculations, and all computed CASSCF states were included on the subsequent XMCQDPT2 calculations. In the XMCQDPT2 calculations, the denominator-energy shift (EDSHIFT $=0.02$ Hartree) and density modification (IFITD5=.T.) were employed. In addition, the excitation spectra of the selected models were computed with the CIS method and the time-dependent DFT method employing the CAM-B3LYP

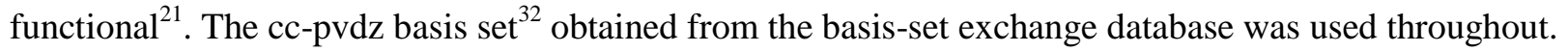

Active-site cluster models. We modeled active sites of several phytochrome proteins using a clustermodel approach. The clusters were comprised of the BV or РФB chromophore covalently bound to the cysteine side chain, several highly conserved residues and water molecules. So far, our models are the largest in size models that treat the phytochrome active site using methods of quantum chemistry. Employing the cluster model approach enables accounting for the tetrapyrrole electronic structure and interactions of tetrapyrroles with their immediate environment at the same level of theory. The starting geometries of the clusters were prepared using the coordinates of the following X-ray crystal structures. The list of all models, model composition and other important characteristics are presented in Table S6. The model of the Pfr state of AtPhyB was prepared not from the respective crystal structure (as the latter has not been determined) but starting from the initial coordinates of the Pr-state AtPhyB cluster by rotating the pyrrole ring $\mathrm{D}$ about the $\mathrm{C} 15-\mathrm{C} 16$ bond and then by moving the Asp307 side chain (corresponding to AspD) closer to ring D by modifying initial values of the $\mathrm{C} \alpha-\mathrm{C} \beta-\mathrm{C} \gamma$ angle and $\mathrm{C} \beta-\mathrm{C} \gamma$ $\mathrm{C} \delta$-O torsion. The hydrogen atoms to the models were added by using the model-building tool of the HyperChem program ${ }^{33}$. The tetrapyrrole chromophore was considered protonated, whereas the carboxylates of the chromophore propionic groups and of AspD were deprotonated. The conserved histidines interacting with pyrrole rings $\mathrm{C}$ and $\mathrm{D}$ were neutral in the $\varepsilon$-form if not stated otherwise. The models with the $\varepsilon$-histidines were used to study the effect of AspD by removing the latter form the cluster. In addition, we considered models in which the protonation state of the histidine interacting with ring D (HisD) was varied to protonated or neutral $\delta$-form. The composition of the cluster models is demonstrated in Figure S7. 
Calculations of the cluster WT models and models w/o AspD. In our study, we considered active-site geometries suggested by experimental crystal structures. To perform subsequent excited-state calculations, the geometries were optimized in Cartesian coordinates starting from the coordinates of the selected pdb structures. As optimization in Cartesian coordinates progresses rather slowly, we opted for performing this optimization for a limited number of step (same number of steps for the models that were compared afterwards) rather than keeping fixed coordinates of selected atoms. Resulting geometries describe local minima on the potential energy surface suggested by the parent crystal structures. As recently demonstrated ${ }^{8}$, optimized geometries reproduce the spectral shifts obtained by performing MD sampling. However, in the case of a chromophore as large as tetrapyrrole, a semiempirical QM method had to be used in order to afford the sampling ${ }^{8}$. In contrast, using optimized geometries of cluster models allows employing rather accurate QM methods. Geometry sampling with classical MD trajectories ${ }^{34}$ does not examine the chromophore electronic structure changes and therefore, is inappropriate for color-tuning studies.

In our study, geometries of models indicated as "cluster WT" (containing HisD in the $\varepsilon$-form) was optimized for 120 steps. During the first 20 steps of optimization, the coordinates of the $\mathrm{H}-\mathrm{N}$ groups in His side chains were frozen. During the following 100 steps, the coordinates of all atoms of the cluster model were optimized. Changes of the energy gradient during optimization are presented for the five cluster WT models in Figure S8. The maximum and RMS gradient values for the geometries at which the excitation energies were computed are listed in Table S7. In order to reveal the effect of AspD, the respective side chain was replaced by the $\mathrm{CH}_{3}$ - group (models w/o AspD) in the cluster WT and after this modification another 20 step of geometry optimization were performed with the PBE0-D3 method in Cartesian coordinates. At these re-optimized geometries, the excited-state energies were recomputed with the XMCQDPT2 method. The geometries, excitation energies and molecular properties were compared for the original cluster WT models and models w/o AspD.

Calculations of isolated chromophores. To evaluate the effect of intermolecular interactions of the tetrapyrrole and its environment in the cluster models, the excitation energies were computed for the tetrapyrrole fragments (the propionic groups PropB and PropC were replaced by the $\mathrm{CH}_{3}$ groups) extracted from the optimized cluster geometries without performing further geometry optimization. These energies were compared with the excitation energies of the tetrapyrroles at their optimized geometries (also the propionic groups PropB and PropC were replaced by the $\mathrm{CH}_{3}$ groups). The latter were obtained by the tetrapyrrole geometry optimization starting from the crystal structure coordinates performed with the PBE0-D3 method in Cartesian coordinates without employing any geometry constrains until the maximum energy gradient was below 0.0001 a.u.

Calculations of the cluster models with protonated and $\delta$ - HisD and w/o HisD. Additional calculations were performed testing the effect of the protonation state of the semi-conserved histidine (HisD) that interacts, directly of via a water molecule with pyrrole ring $\mathrm{D}$ in the Pr state and with the propionate bound to the pyrrole ring $\mathrm{C}$ (PropC) in the Pfr state. The models containing HisD in the $\delta$-form $(\delta \mathrm{HisD})$ or protonated $(\mathrm{HisD}(\mathrm{H}))$ were prepared and their geometry was optimized in a similar manner as the geometry of the cluster WT models containing the $\varepsilon$-form ( $\varepsilon H i s D)$. Models indicated as w/o HisD were prepared by removing the HisD ( $\varepsilon$-form) residue from the optimized WT models and then performing 20 steps of geometry optimization in Cartesian coordinates. The excited-state energies of all 
these models were recomputed with the XMCQDPT2 method. As our cluster model Pfr AtPhyB did not contain HisD (Figure S7), the effect of this residue was not investigated for this case.

\section{S1.2. Molecular dynamics (MD) simulations}

Protein models. The MD simulations were performed for the Pr and Pfr states of the DrBphP and Pfr state of the PaBphP proteins. Table S8 presents the list of all simulations performed. The initial atomic coordinates were taken from the crystal structures ${ }^{9-13}$. The crystallographic water molecules were preserved in the models. A water molecule, corresponding to the pyrrole water, was not resolved in the parent $4 \mathrm{o} 0 \mathrm{p}$ structure $^{11}$, therefore it was added from the $2 \mathrm{o} 9 \mathrm{c}$ structure $^{9}$ after the overlay of both structures. The missing residues were added using the protein-building module of the HyperChem software $^{33}$, and the missing side chains were added using the VMD software version 1.9.2a33 ${ }^{35}$. Standard protonation states of the titratable residues corresponding to $\mathrm{pH}=7$ were considered, except for the histidine HisD at the tetrapyrrole binding site. Whereas the histidines in the protein were considered neutral in the $\mathrm{N} \varepsilon-\mathrm{H}$ form, HisD, which is semi-conserved in bacteriophytochromes, was considered in various protonation states (Table S8). Choosing HisD protonated stabilized several hydrogen bonds around tetrapyrrole in our MD simulations as demonstrated in Figure S9. Interestingly, introducing protonated $\mathrm{HisD}(\mathrm{H})$ in our cluster models resulted in an increased $\mathrm{Pr} / \mathrm{Pfr}$ red shift by further decreasing the $\mathrm{S}_{0}-\mathrm{S}_{1}$ energy of the Pfr models (the respective energies are presented in Table $\mathrm{S} 1$ ). The protein construct of interest was solvated in a cubic water box using the solvate package of the VMD software by introducing a $15-\AA$ layer of water molecules and $150 \mathrm{mM}$ of sodium cations with the appropriate amount of the chloride anions to neutralize the system.

Special wild-type models with protonated biliverdin 15Ea configuration but with different conformation of CBD and PHY domains of DrBphP (Table S8) were prepared from the PrDr-CBDPHY model with protonated HisD. 15E-CBD(Pr)PHY(Pr) model was prepared from the solvated PrDr-CBDPHY model, coordinates after $5 \mathrm{~ns}$ of equilibration taking as initial coordinates and then manually rotating the ring D of the biliverdin. In contrast to all other models, this model was not subjected to equilibration run but directly to the production run. For $15 \mathrm{E}-\mathrm{CBD}(\mathrm{Pr})$ model, the coordinates of the CBD domain and solvent (water and ions) atoms within $4 \AA$ were taken after $5 \mathrm{~ns}$ of equilibration of the solvated PrDr-CBDPHY model, then biliverdin ring $\mathrm{D}$ was rotated and protein was additionally solvated as described above. To prepare 15E-CBD(Pr)PHY(Pfr) model, the coordinates of the Dr-Pr-CBDPHY model after 5 ns of equilibration (for CBD domain coordinates) and the $5 \mathrm{c} 5 \mathrm{k}$ structure was aligned (for PHY domain coordinates) and appropriate coordinates were used. The biliverdin ring $\mathrm{D}$ was rotated and protein was solvated as described above.

Force field parameters. The CHARMM22 parameters ${ }^{36,37}$ were used for the protein in combination with the TIP3 water parameters. The published CHARMM22 parameters for biliverdin ${ }^{38,}{ }^{39}$ were used for 15Za-biliverdin, whereas these parameters were slightly modified for 15Ea-biliverdin as indicated in Table S9 and Figure S10.

Software and MD protocol. Simulations were performed using the NAMD software version 2.9 (NAMD version 2.11 and 2.12$)^{27}$. The solvated protein was subjected to 20000 steps of energy minimization followed by $5 \mathrm{~ns}$ of equilibration at $300 \mathrm{~K}$. During the equilibration, all atoms of the protein backbone present in the pdb model and the oxygen of the pyrrole water were constrained using harmonic potentials. Following equilibration, the production run was performed for $80 \mathrm{~ns}$ at $300 \mathrm{~K}$. The equilibration and 
production runs were performed with the 2 fs integration step in the NPT ensemble using a combination of Langevin dynamics ${ }^{40}$ and Nosé-Hoover thermostat ${ }^{41}$. The trajectories were analyzed using Trajectory Tools of the VMD software. Distributions of the distances were plotted from the last $70 \mathrm{~ns}$ of the $80 \mathrm{~ns}$ simulation.

\section{S2 Supplementary figures}

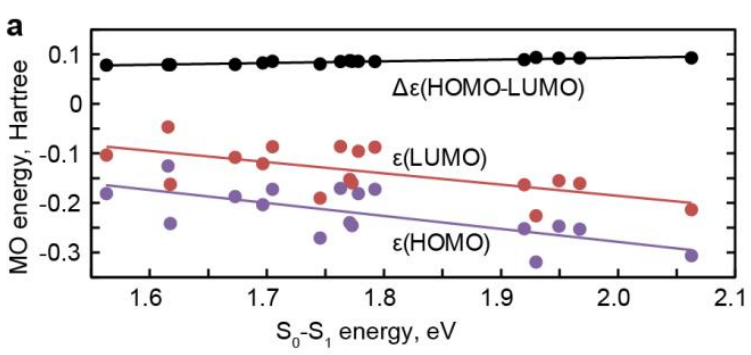

b
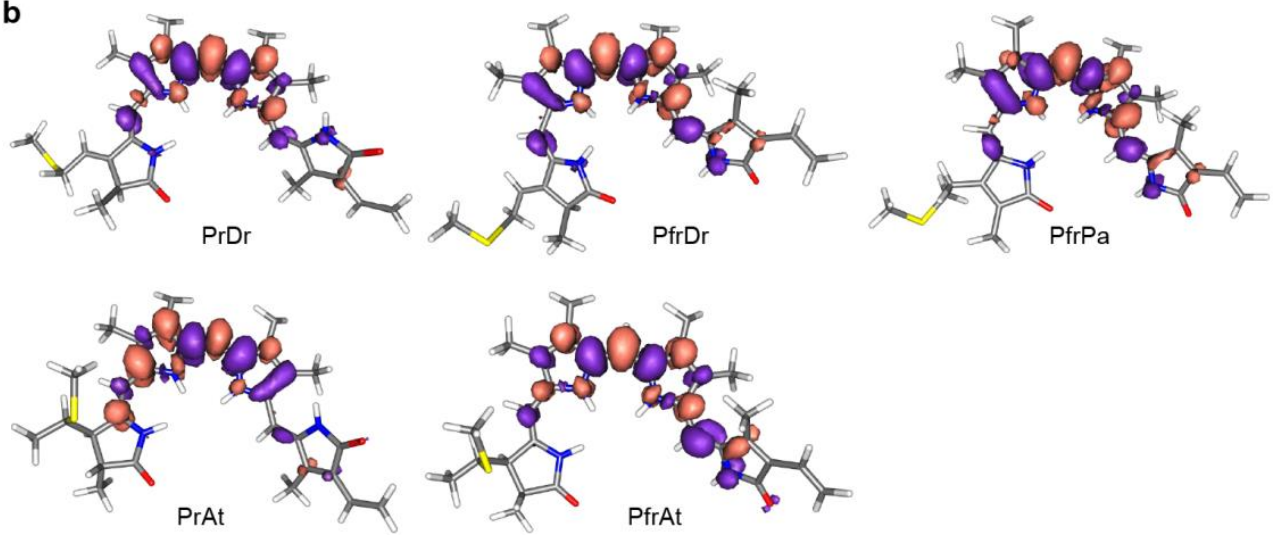

Figure S1. Single-electron excitation character of the $S_{0}-S_{1}$ transition of linear tetrapyrroles in phytochrome models. (a) Correlation between the $\mathrm{S}_{0}-\mathrm{S}_{1}$ energies (XMCQDPT2) and the HOMO and LUMO energies or difference energies (PBE0) in all computed cluster models characterized in Table S1. (b) Electron-density changes upon the $\mathrm{S}_{0}-\mathrm{S}_{1}$ transition $\left(\mathrm{S}_{1}\right.$-state minus $\mathrm{S}_{0}$-state density; purple and orange indicate negative and positive density, respectively). The density difference is demonstrated for the cluster WT models. For clarity, only the tetrapyrrole molecular structure without propionates is shown in the Figure. 

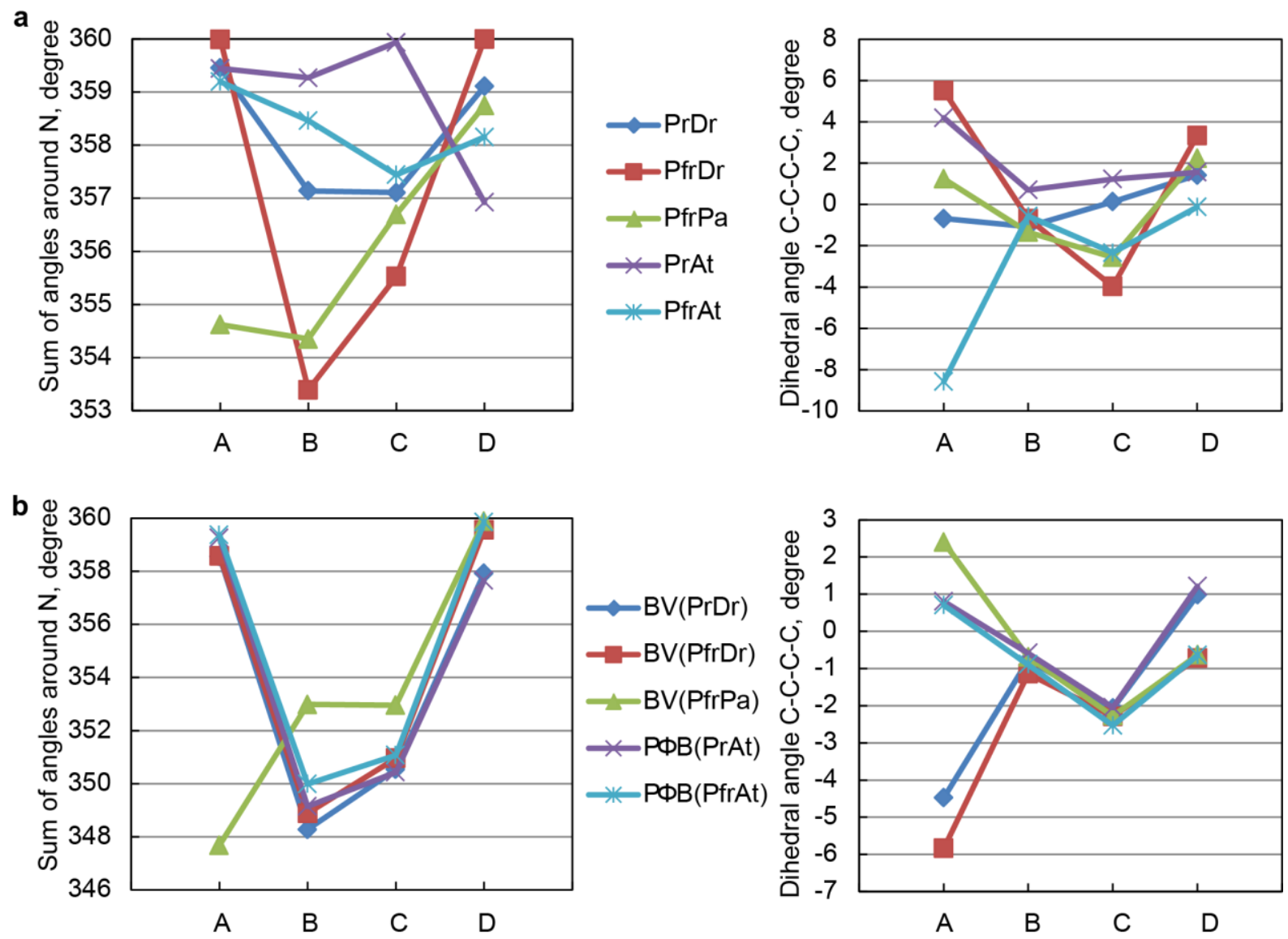

Figure S2. Planarity of pyrrole rings in the phytochrome models (a) and optimized chromophores (b).
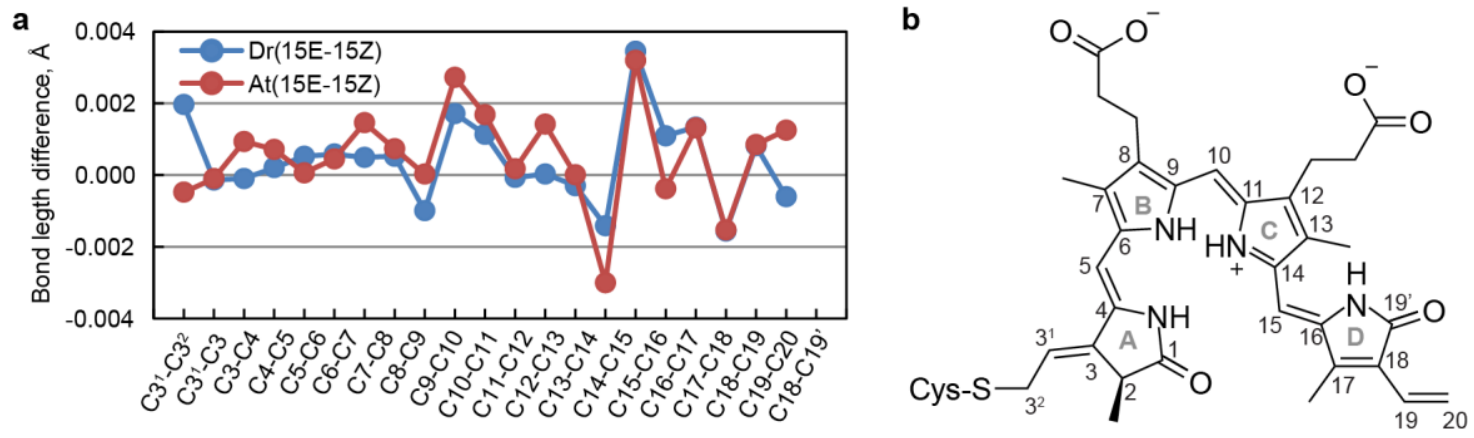

Figure S3. Bond-length comparison in the tetrapyrrole 15Ea and 15Za configurations. (a) Differences of bond length. The positive and negative differences indicate a longer and shorter bond length, respectively, in the $15 \mathrm{Ea}$ configuration as compared to the $15 \mathrm{Za}$ configuration of the biliverdin (Dr, shown in blue) and phytochromobilin (At, shown in red) chromophores. The geometry was optimized with the PBE0-D3/cc-pvdz method for isolated chromophores starting from the coordinates of the crystal structures 2o9c and 5c5k (Dr), and 4our (At). (b) Labelling of the tetrapyrrole atoms presented for the biliverdin chromophore in the 15Za configuration. 

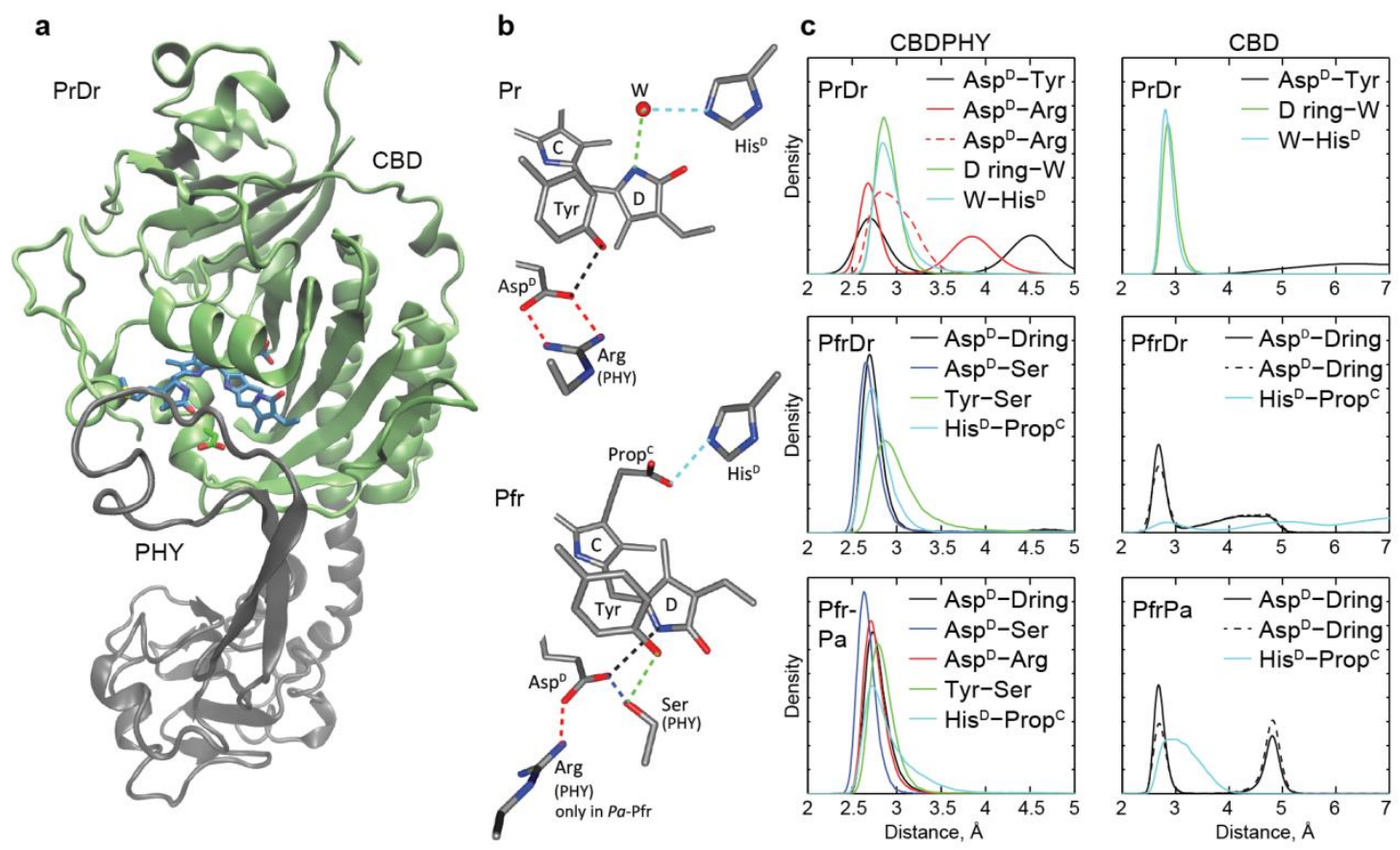

d PrDr-CBDPHY
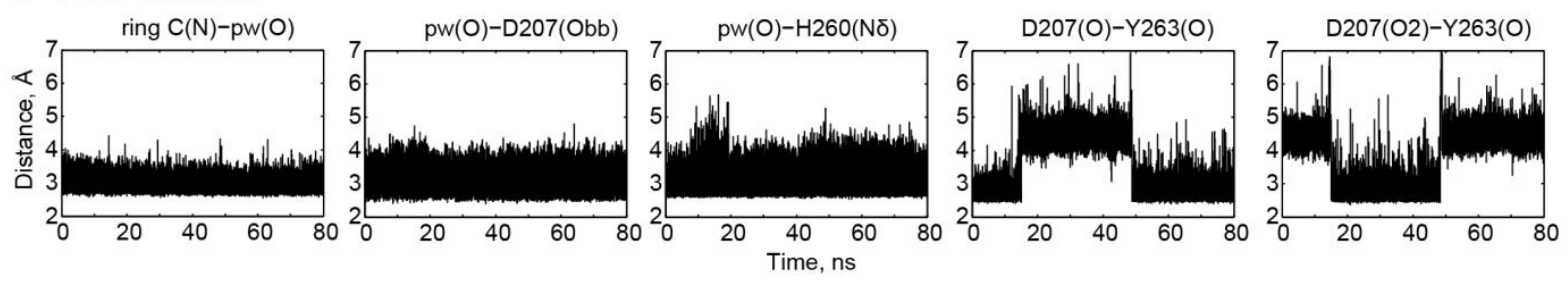

e PrDr-CBD
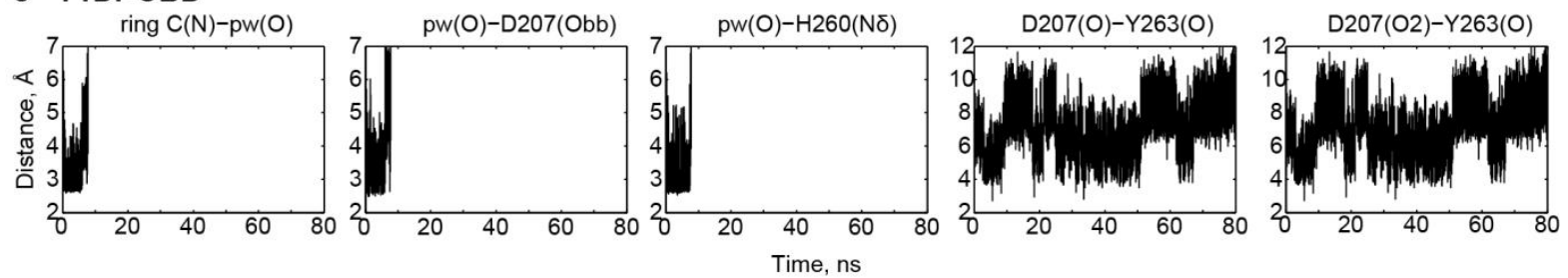

Figure S4. Comparison of the CBD and CBDPHY models of the Pr- and Pfr-states of DrBphP and Pfr-state of PaBphP. (a) A cartoon representation of the phytochrome domain structure. The CBD (shown in green) and PHY (shown in gray) domains comprise the phytochrome photosensory module the minimum-length protein construct forming the Pfr state. Using the stick representation, the tetrapyrrole cofactor and conserved aspartate (AspD) are indicated. (b) Interactions of the tetrapyrrole ring $\mathrm{D}$ in the $\mathrm{Pr}$ and $\mathrm{Pfr}$ states. (c) Comparison of the distances characterizing hydrogen-bonding interactions in the CBDPHY and CBD proteins. The side chains and water molecule for which the distances are presented are illustrated in panel b. (d) Interaction of pyrrole water ( $\mathrm{pw}$ ) with the active-site residues and the hydrogen bond between Asp207(AspD) and Tyr263 in the Pr state of the DrBphP CBDPBY protein. (e) Exchange of pyrrole water (pw) and opening and closing of the Asp207-Tyr263 gate in the truncated Pr DrBphP CBD protein. 

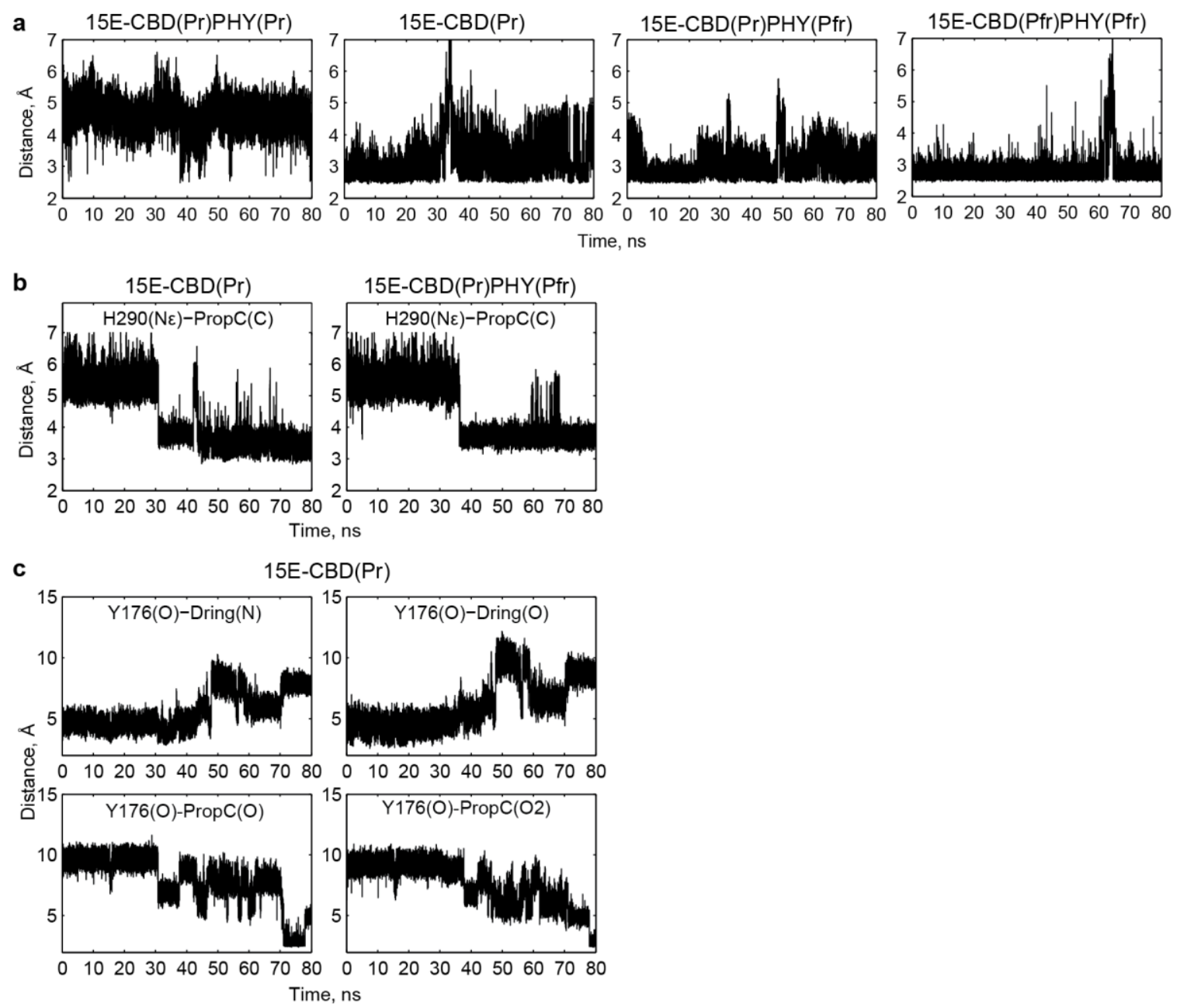

Figure S5. Hydrogen-bond dynamics induced by biliverdin isomerization from 15Za to 15Ea. (a) The distance between Asp207 (AspD) and 15Ea ringD in the series of protein constructs modelling formation of the AspD-Ring D hydrogen bond after ring D isomerization. (b) Enhanced interactions between His290 (HisD) and propionate of ring C (PropC) after isomerization of ring D (15Ea) inside the Pr CBD domain. The interactions were facilitated upon truncation of the Pr PHY domain or addition of the Pfr PHY domain. (c) Unstable interactions of Tyr176 with the isomerized ring D (15Ea) inside the Pr CBD protein in the absence of the PHY domain. All simulations were performed for the DrBphP protein.

a

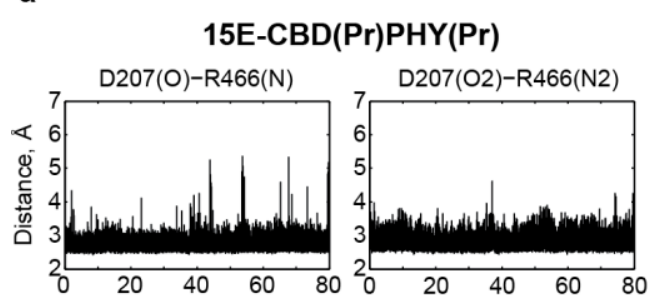

b

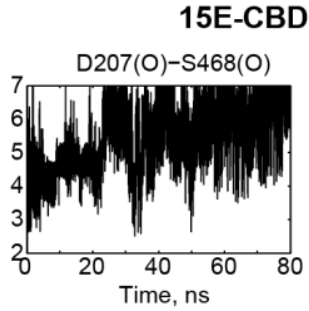

C 15E-CBD(Pfr)PHY(Pfr)

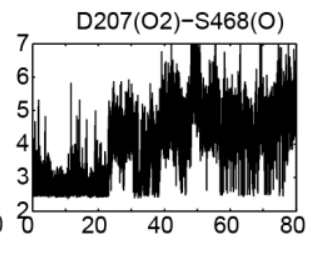

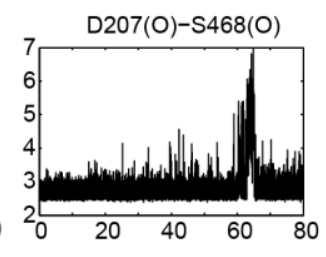

Figure S6. AspD interactions with the conserved arginine of Pr-PHY and conserved serine of PfrPHY domain in the DrBphP models with 15Ea-biliverdin. (a) AspD-Arg interactions in the 15E$\mathrm{CBD}(\mathrm{Pr}) \mathrm{PHY}(\mathrm{Pr})$ model. (b) AspD-Ser interactions in the 15E-CBD(Pr)PHY(Pfr) model. (c) AspD-Ser interactions in the 15E-CBD(Pfr)PHY(Pfr) model. 

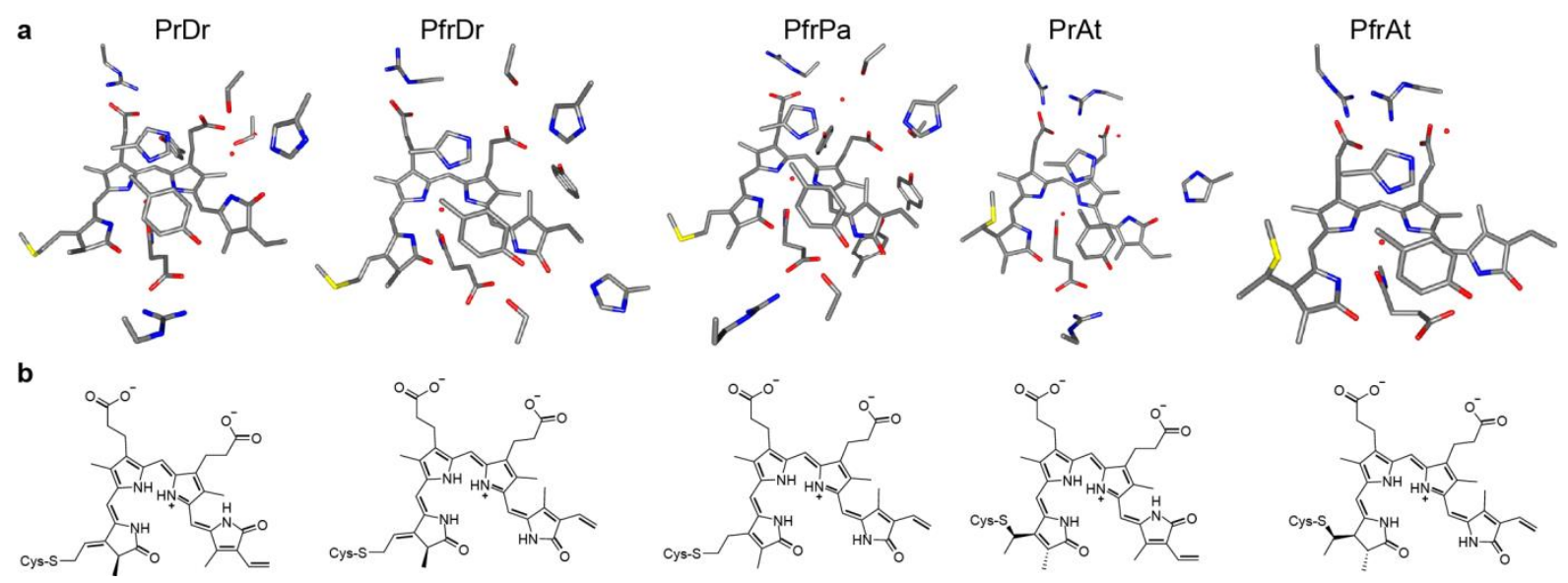

Figure S7. Composition of the cluster models. (a) Models of the five phytochrome active sites considered in the study. Hydrogen atoms are omitted for clarity. The residues and water molecules included in the models are specified in Table S6. (b) Chemical structure of the linear tetrapyrrole chromophores in the corresponding cluster models shown in panel a.
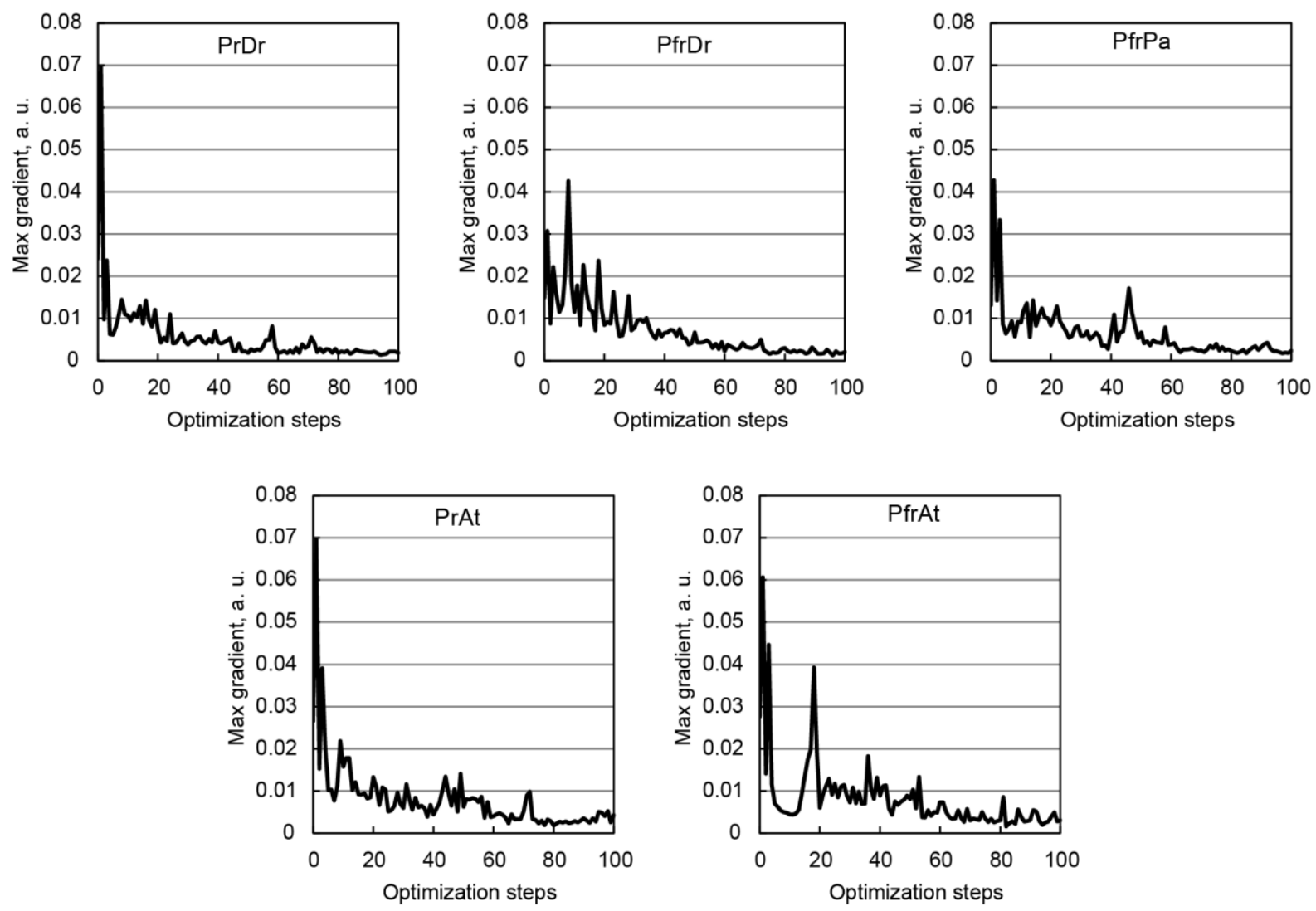

Figure S8. Energy gradients of the WT cluster models containing \&HisD. 
neutral $\delta$
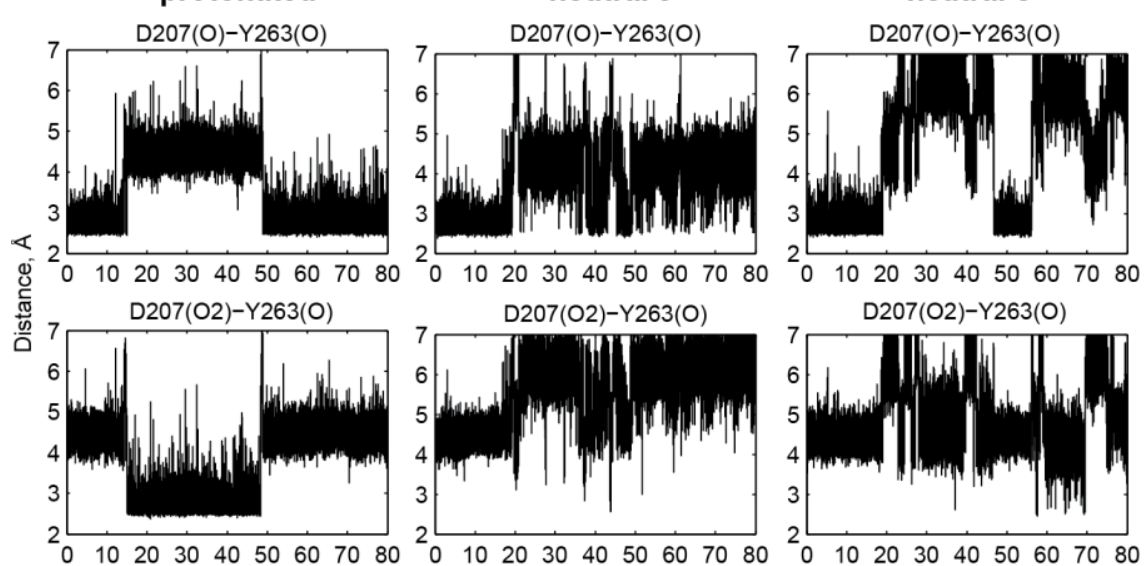

D207(O2)-Y263(O)

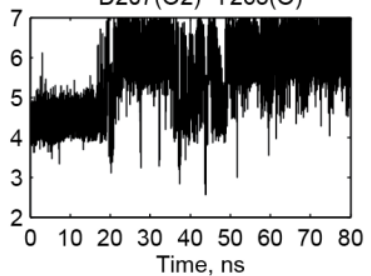

D207(O2)-Y263(O)

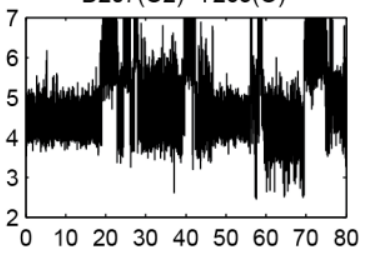

b

protonated

neutral $\varepsilon$

$\mathrm{H} 290(\mathrm{~N} \varepsilon)-\operatorname{PropC}(\mathrm{O})$

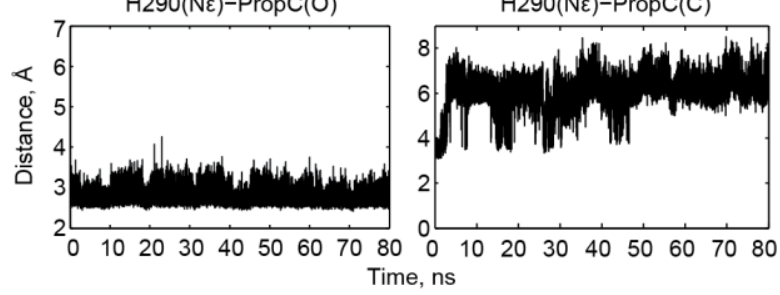

d

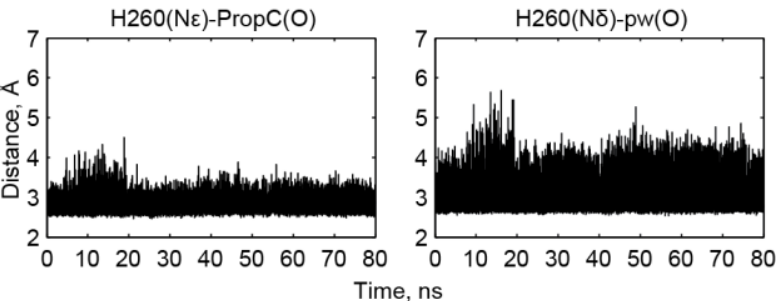

c

neutral $\varepsilon$
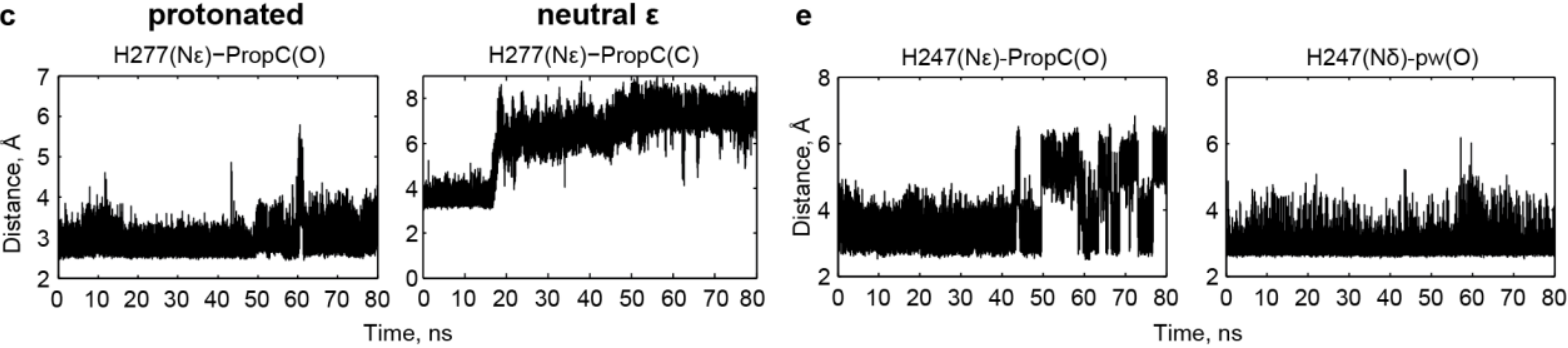

Figure S9. Stabilization of hydrogen bonds at the active site by protonated HisD. (a) Interactions of Tyr263 and Asp207(AspD) in Pr DrBphP CBDPHY depending on the protonation state of His290 (HisD) (indicated above the plots). (b) Interactions of the His290 (HisD) with the propionate of ring C (PropC) in Pfr DrBphP CBDPHY depending on the His290 protonation state (indicated above the plots). (c) Interactions of His277 (HisD) with the propionate of ring $\mathrm{C}$ (PropC) in Pfr PaBphP CBDPHY depending on the His277 protonation state (indicated above the plots). (d) Stable interactions of His260 with the propionate of ring $\mathrm{C}$ (PropC) and pyrrole water in Pr DrBphP CBDPHY in the presence of protonated His290 (HisD). (e) Stable interactions of His247 with the propionate of ring C (PropC) and pyrrole water in Pfr PaBphP CBDPHY in the presence of protonated His277 (HisD). 

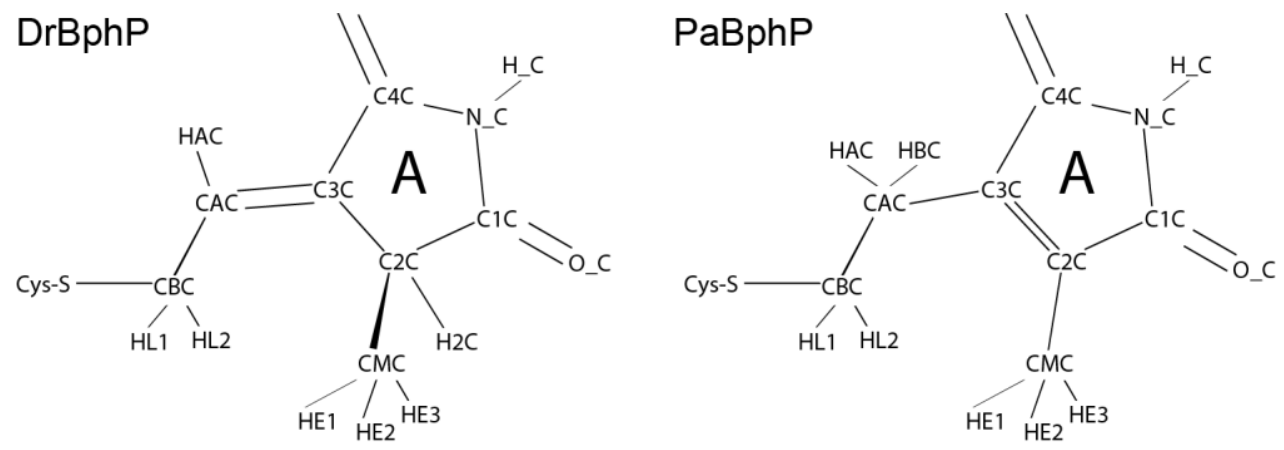

Figure S10. Atom names in ring A of biliverdin bound to DrBphP and PaBphP used for the force field parameters preparation. 


\section{S3. Supplementary tables}

Table S1. Excitation energies, molecular-orbital energies and dipole moments computed for the activesite and tetrapyrrole chromophore models. In brackets, oscillator strength of electronic transitions are indicated. For comparison, the energies corresponding to the Q-band maximum of the respective proteins are given.

\begin{tabular}{|c|c|c|c|c|c|}
\hline & Pr DrBphP & Pfr DrBphP & Pfr PaBphP & Pr AtPhyB & Pfr AtPhyB \\
\hline $\begin{array}{l}\text { Exp. Q-max. } \\
(\mathrm{eV})\end{array}$ & $1.77^{19}$ & $1.65^{19}$ & $1.65^{20}$ & $1.87^{13}$ & $1.71^{13}$ \\
\hline $\begin{array}{l}\text { Exp. Soret-max. } \\
(\mathrm{eV})\end{array}$ & 3.15 & 3.03 & 3.03 & 3.24 & 3.09 \\
\hline \multicolumn{6}{|c|}{ WT cluster models (containing EHisD and AspD), XMCQDPT2 method } \\
\hline $\mathrm{S}_{0}-\mathrm{S}_{1}(\mathrm{eV})$ & $1.78(1.323)$ & $1.62(1.214)$ & $1.67(1.091)$ & $1.97(1.243)$ & $1.71(1.360)$ \\
\hline $\mathrm{S}_{0^{-}} \mathrm{S}_{2}$ & $2.74(0.022)$ & $2.53(0.036)$ & $2.51(0.016)$ & $2.98(0.005)$ & $2.59(0.034)$ \\
\hline $\mathrm{S}_{0}-\mathrm{S}_{3}$ & $3.00(0.038)$ & $2.61(0.067)$ & $2.66(0.082)$ & $3.50(0.701)$ & $2.80(0.071)$ \\
\hline $\mathrm{S}_{0}-\mathrm{S}_{4}$ & $3.25(0.487)$ & $2.99(0.380)$ & $2.97(0.461)$ & $3.67(0.074)$ & $3.16(0.330)$ \\
\hline $\mathrm{S}_{0}-\mathrm{S}_{5}$ & $3.71(0.044)$ & $3.61(0.043)$ & $3.57(0.071)$ & $4.29(0.054)$ & $3.65(0.006)$ \\
\hline $\mathrm{S}_{0}-\mathrm{S}_{6}$ & $4.09(0.096)$ & $3.80(0.097)$ & $3.80(0.074)$ & $4.43(0.288)$ & $3.93(0.135)$ \\
\hline $\mathrm{d}_{0}$, Debye & 30.5 & 40.4 & 37.2 & 23.7 & 47.2 \\
\hline $\mathrm{d}_{1}$, Debye & 32.7 & 41.5 & 39.8 & 20.3 & 45.4 \\
\hline$\left|\mathrm{d}_{1}\right|-\left|\mathrm{d}_{0}\right|$, Debye & 2.14 & 1.09 & 2.62 & -3.38 & -1.82 \\
\hline$\angle\left(\mathrm{d}_{0}, \mathrm{~d}_{1}\right)$, ged. & 1.3 & 3.2 & 1.9 & 6.2 & 0.4 \\
\hline \multicolumn{6}{|l|}{ PBE0 method } \\
\hline$\varepsilon(\mathrm{HOMO})$, a.u. & -0.1816 & -0.1258 & -0.1873 & -0.2535 & -0.1729 \\
\hline$\varepsilon($ LUMO), a.u & -0.0961 & -0.0471 & -0.1081 & -0.1611 & -0.0870 \\
\hline \multicolumn{6}{|c|}{ Cluster models w/o AspD (containing \&HisD), XMCQDPT2 method } \\
\hline $\mathrm{S}_{0}-\mathrm{S}_{1}$ & $1.77(1.224)$ & $1.70(1.188)$ & $1.75(1.028)$ & $1.93(1.254)$ & $1.92(1.297)$ \\
\hline $\mathrm{S}_{0}-\mathrm{S}_{2}$ & $2.62(0.005)$ & $2.57(0.007)$ & $2.51(0.018)$ & $2.86(0.006)$ & $2.82(0.009)$ \\
\hline $\mathrm{S}_{0}-\mathrm{S}_{3}$ & $2.84(0.007)$ & $3.10(0.151)$ & $3.12(0.453)$ & $3.11(0.239)$ & $3.46(0.668)$ \\
\hline $\mathrm{S}_{0}-\mathrm{S}_{4}$ & $3.31(0.483)$ & $3.26(0.497)$ & $3.35(0.489)$ & $3.43(0.347)$ & $3.54(0.059)$ \\
\hline $\mathrm{S}_{0}-\mathrm{S}_{5}$ & $3.62(0.028)$ & $3.63(0.031)$ & $3.87(0.028)$ & $3.91(0.011)$ & $4.18(0.128)$ \\
\hline $\mathrm{S}_{0}-\mathrm{S}_{6}$ & $4.08(0.000)$ & $4.09(0.080)$ & $4.12(0.051)$ & $4.25(0.015)$ & $4.39(0.149)$ \\
\hline$\left|\mathrm{d}_{1}\right|-\left|\mathrm{d}_{0}\right|$, Debye & 1.80 & 0.29 & 2.09 & -2.30 & -1.11 \\
\hline \multicolumn{6}{|l|}{ PBE0 method } \\
\hline$\varepsilon(\mathrm{HOMO})$ & -0.2460 & -0.2038 & -0.2710 & -0.3196 & -0.2526 \\
\hline$\varepsilon($ LUMO $)$ & -0.1601 & -0.1213 & -0.1906 & -0.2262 & -0.1636 \\
\hline \multicolumn{6}{|c|}{ WT cluster models (containing \&HisD and AspD), TD-CAM-B3LYP method } \\
\hline $\mathrm{S}_{0}-\mathrm{S}_{1}$ & $2.15(0.975)$ & $1.98(0.845)$ & $1.97(0.622)$ & $2.32(0.883)$ & $2.14(0.883)$ \\
\hline $\mathrm{S}_{0}-\mathrm{S}_{2}$ & $3.05(0.001)$ & $2.55(0.049)$ & $2.44(0.000)$ & $3.26(0.002)$ & $2.54(0.051)$ \\
\hline $\mathrm{S}_{0}-\mathrm{S}_{3}$ & $3.25(0.025)$ & $3.18(0.016)$ & 2.81(0.016) & $3.26(0.001)$ & $3.35(0.131)$ \\
\hline $\mathrm{S}_{0}-\mathrm{S}_{4}$ & $3.35(0.192)$ & $3.21(0.019)$ & 2.92(0.002) & $3.51(0.124)$ & $3.40(0.086)$ \\
\hline $\mathrm{S}_{0}-\mathrm{S}_{5}$ & $3.53(0.129)$ & $3.27(0.384)$ & $3.18(0.075)$ & $3.70(0.548)$ & $3.47(0.087)$ \\
\hline $\mathrm{S}_{0}-\mathrm{S}_{6}$ & $3.57(0.067)$ & $3.42(0.009)$ & $3.29(0.054)$ & $3.71(0.011)$ & $3.59(0.037)$ \\
\hline $\mathrm{S}_{0}-\mathrm{S}_{7}$ & $3.61(0.242)$ & $3.47(0.028)$ & $3.36(0.879)$ & $3.78(0.038)$ & $3.62(0.239)$ \\
\hline $\mathrm{S}_{0}-\mathrm{S}_{8}$ & $3.64(0.047)$ & $3.48(0.365)$ & $3.49(0.015)$ & $3.93(0.194)$ & $3.77(0.013)$ \\
\hline
\end{tabular}




\begin{tabular}{|c|c|c|c|c|c|}
\hline $\mathrm{S}_{0^{-}} \mathrm{S}_{9}$ & $3.67(0.255)$ & $3.56(0.000)$ & $3.54(0.043)$ & $4.01(0.003)$ & $3.78(0.028)$ \\
\hline $\mathrm{S}_{0}-\mathrm{S}_{10}$ & $3.96(0.013)$ & $3.65(0.061)$ & $3.72(0.030)$ & $4.14(0.014)$ & $3.83(0.081)$ \\
\hline \multicolumn{6}{|c|}{ WT cluster models (containing EHisD and AspD), CIS method } \\
\hline $\mathrm{S}_{0}-\mathrm{S}_{1}$ & $2.62(1.321)$ & $2.48(1.225)$ & $2.45(0.891)$ & $\mathrm{n} / \mathrm{c}$ & $\mathrm{n} / \mathrm{c}$ \\
\hline $\mathrm{S}_{0}-\mathrm{S}_{2}$ & $4.40(1.455)$ & $4.23(1.482)$ & $4.17(1.854)$ & & \\
\hline $\mathrm{S}_{0}-\mathrm{S}_{3}$ & $4.81(0.013)$ & $4.43(0.064)$ & $4.46(0.093)$ & & \\
\hline $\mathrm{S}_{0}-\mathrm{S}_{4}$ & $4.84(0.224)$ & $4.56(0.034)$ & $4.67(0.031)$ & & \\
\hline $\mathrm{S}_{0}-\mathrm{S}_{5}$ & $5.01(0.020)$ & 4.82(0.010) & 4.71(0.003) & & \\
\hline $\mathrm{S}_{0}-\mathrm{S}_{6}$ & $5.19(0.062)$ & $4.90(0.081)$ & 4.72(0.079) & & \\
\hline $\mathrm{S}_{0}-\mathrm{S}_{7}$ & $5.28(0.074)$ & $5.11(0.096)$ & $4.85(0.009)$ & & \\
\hline $\mathrm{S}_{0}-\mathrm{S}_{8}$ & $5.39(0.002)$ & $5.34(0.022)$ & $5.18(0.287)$ & & \\
\hline $\mathrm{S}_{0}-\mathrm{S}_{9}$ & $5.42(0.014)$ & $5.47(0.000)$ & $5.23(0.002)$ & & \\
\hline $\mathrm{S}_{0}-\mathrm{S}_{10}$ & $5.47(0.049)$ & $5.51(0.094)$ & $5.32(0.009)$ & & \\
\hline \multicolumn{6}{|c|}{ Tetrapyrrole chromophore at the WT cluster geometry, XMCQDPT2 method } \\
\hline $\mathrm{S}_{0}-\mathrm{S}_{1}$ & $1.85(1.368)$ & $1.73(1.237)$ & $1.73(1.119)$ & $2.02(1.312)$ & $1.87(1.367)$ \\
\hline $\mathrm{S}_{0}-\mathrm{S}_{2}$ & $2.73(0.040)$ & $2.51(0.017)$ & $2.48(0.020)$ & $2.91(0.039)$ & $2.66(0.008)$ \\
\hline $\mathrm{S}_{0}-\mathrm{S}_{3}$ & $3.06(0.044)$ & $2.95(0.064)$ & $2.83(0.070)$ & $3.38(0.071)$ & $3.04(0.084)$ \\
\hline $\mathrm{S}_{0}-\mathrm{S}_{4}$ & $3.43(0.480)$ & $3.26(0.555)$ & $3.20(0.586)$ & $3.69(0.573)$ & $3.35(0.420)$ \\
\hline $\mathrm{S}_{0}-\mathrm{S}_{5}$ & $3.89(0.075)$ & $3.80(0.031)$ & $3.76(0.047)$ & $4.29(0.062)$ & $3.93(0.044)$ \\
\hline $\mathrm{S}_{0}-\mathrm{S}_{6}$ & $4.03(0.093)$ & $3.96(0.084)$ & $3.83(0.064)$ & $4.39(0.418)$ & 4.01(0.138) \\
\hline \multicolumn{6}{|c|}{ Tetrapyrrole chromophore at the optimized geometry, XMCQDPT2 method } \\
\hline $\mathrm{S}_{0}-\mathrm{S}_{1}$ & $1.68(1.042)$ & $1.64(1.006)$ & $1.66(0.908)$ & $1.74(1.079)$ & $1.68(1.012)$ \\
\hline $\mathrm{S}_{0}-\mathrm{S}_{2}$ & $2.64(0.062)$ & $2.58(0.052)$ & $2.53(0.050)$ & $2.69(0.056)$ & $2.62(0.041)$ \\
\hline $\mathrm{S}_{0}-\mathrm{S}_{3}$ & $2.88(0.035)$ & $2.84(0.046)$ & $2.70(0.065)$ & $2.92(0.043)$ & $2.84(0.058)$ \\
\hline $\mathrm{S}_{0}-\mathrm{S}_{4}$ & $3.33(0.529)$ & $3.25(0.557)$ & $3.20(0.616)$ & $3.40(0.516)$ & $3.30(0.567)$ \\
\hline $\mathrm{S}_{0}-\mathrm{S}_{5}$ & $3.74(0.156)$ & $3.68(0.089)$ & $3.68(0.105)$ & $3.90(0.125)$ & $3.81(0.056)$ \\
\hline $\mathrm{S}_{0}-\mathrm{S}_{6}$ & $3.95(0.107)$ & $3.88(0.103)$ & $3.78(0.057)$ & $3.99(0.119)$ & $3.89(0.144)$ \\
\hline \multicolumn{6}{|c|}{ Protonated HisD: WT cluster models (containing HisD(H) and AspD), XMCQDPT2 method } \\
\hline $\mathrm{S}_{0}-\mathrm{S}_{1}$ & $1.77(1.286)$ & $1.56(1.176)$ & $1.62(1.094)$ & $2.06(1.199)$ & $\mathrm{n} / \mathrm{c}$ \\
\hline $\mathrm{S}_{0}-\mathrm{S}_{2}$ & $2.76(0.000)$ & $2.46(0.040)$ & $2.49(0.025)$ & $2.87(0.097)$ & \\
\hline $\mathrm{S}_{0}-\mathrm{S}_{3}$ & $3.20(0.036)$ & $2.75(0.093)$ & $2.73(0.061)$ & $3.39(0.549)$ & \\
\hline $\mathrm{S}_{0}-\mathrm{S}_{4}$ & $3.35(0.684)$ & $3.09(0.342)$ & $3.03(0.466)$ & $3.75(0.117)$ & \\
\hline $\mathrm{S}_{0}-\mathrm{S}_{5}$ & $3.66(0.001)$ & $3.45(0.017)$ & $3.52(0.050)$ & $4.25(0.211)$ & \\
\hline $\mathrm{S}_{0}-\mathrm{S}_{6}$ & $4.09(0.116)$ & $3.94(0.111)$ & $3.90(0.084)$ & $4.48(0.026)$ & \\
\hline$\left|\mathrm{d}_{1}\right|-\left|\mathrm{d}_{0}\right|$, Debye & -0.04 & -2.01 & 0.69 & 3.10 & \\
\hline \multicolumn{6}{|l|}{ PBE0 method } \\
\hline$\varepsilon(\mathrm{HOMO})$ & -0.2400 & -0.1816 & -0.2418 & -0.3066 & \\
\hline$\varepsilon($ LUMO) & -0.1528 & -0.1035 & -0.1629 & -0.2141 & \\
\hline \multicolumn{6}{|c|}{ Neutral HisD ( $\delta$-form): cluster WT model (containing $\delta$ HisD and AspD), XMCQDPT2 method } \\
\hline $\mathrm{S}_{0}-\mathrm{S}_{1}$ & $1.76(1.335)$ & $\mathrm{n} / \mathrm{c}$ & $\mathrm{n} / \mathrm{c}$ & $\mathrm{n} / \mathrm{c}$ & $\mathrm{n} / \mathrm{c}$ \\
\hline $\mathrm{S}_{0}-\mathrm{S}_{2}$ & $2.71(0.046)$ & & & & \\
\hline $\mathrm{S}_{0}-\mathrm{S}_{3}$ & $3.13(0.432)$ & & & & \\
\hline $\mathrm{S}_{0}-\mathrm{S}_{4}$ & $3.65(0.113)$ & & & & \\
\hline $\mathrm{S}_{0}-\mathrm{S}_{5}$ & $4.08(0.004)$ & & & & \\
\hline $\mathrm{S}_{0}-\mathrm{S}_{6}$ & $4.38(0.043)$ & & & & \\
\hline$\left|\mathrm{d}_{1}\right|-\left|\mathrm{d}_{0}\right|$, Debye & 1.93 & & & & \\
\hline \multicolumn{6}{|l|}{ PBE0 method } \\
\hline$\varepsilon(\mathrm{HOMO})$ & -0.1711 & & & & \\
\hline
\end{tabular}




\begin{tabular}{|l|l|l|l|l|l|}
\hline$\varepsilon($ LUMO) & -0.0861 & & & & \\
\hline \multicolumn{2}{|l|}{ Cluster models w/o HisD (containing AspD), XMCQDPT2 method } \\
\hline $\mathrm{S}_{0}-\mathrm{S}_{1}$ & $1.79(1.341)$ & $\mathrm{n} / \mathrm{c}$ & $\mathrm{n} / \mathrm{c}$ & $1.95(1.244)$ & $\mathrm{n} / \mathrm{c}$ \\
\hline $\mathrm{S}_{0}-\mathrm{S}_{2}$ & $2.72(0.026)$ & & & $2.99(0.005)$ & \\
\hline $\mathrm{S}_{0}-\mathrm{S}_{3}$ & $2.95(0.042)$ & & & $3.52(0.573)$ & \\
\hline $\mathrm{S}_{0}-\mathrm{S}_{4}$ & $3.24(0.468)$ & & & $3.64(0.152)$ & \\
\hline $\mathrm{S}_{0}-\mathrm{S}_{5}$ & $3.74(0.047)$ & & & $4.28(0.005)$ & \\
\hline $\mathrm{S}_{0}-\mathrm{S}_{6}$ & $4.08(0.101)$ & & & $4.42(0.409)$ & \\
\hline$\left|\mathrm{d}_{1}-\right| \mathrm{d}_{0} \mid$, Debye & 3.07 & & & -2.72 & \\
\hline PBE0 method & & & & & \\
\hline$\varepsilon($ HOMO) & -0.1729 & & & -0.2475 & \\
\hline$\varepsilon($ LUMO) & -0.0878 & & & -0.1553 & \\
\hline
\end{tabular}

$\mathrm{n} / \mathrm{c}-$ not computed.

Table S2. Dihedral angles of the tetrapyrrole methine bridges in the WT cluster models containing $\varepsilon$ HisD, tetrapyrrole chromophores at the optimized geometries and starting geometries.

\begin{tabular}{|c|c|c|c|c|c|}
\hline & Pr DrBphP & Pfr DrBphP & Pfr PaBphP & Pr AtPhyB & Pfr AtPhyB \\
\hline \multicolumn{6}{|c|}{ Dihedral angle (degree) } \\
\hline \multicolumn{6}{|l|}{ WT cluster models } \\
\hline C3-C4-C5-C6 & -179.7 & -167.5 & -175.5 & -176.4 & 177.7 \\
\hline C4-C5-C6-C7 & -164.8 & -162.4 & -162.5 & -173.8 & 176.1 \\
\hline C8-C9-C10-C11 & 175.5 & 174.2 & 166.0 & -170.7 & -174.5 \\
\hline C9-C10-C11-C12 & 179.3 & 167.3 & 169.8 & -178.4 & 171.8 \\
\hline C13-C14-C15-C16 & 41.4 & 27.0 & 31.2 & 43.4 & 34.6 \\
\hline C14-C15-C16-C17 & -164.0 & 29.0 & 31.2 & -174.5 & 26.7 \\
\hline \multicolumn{6}{|c|}{ Tetrapyrrole chromophores at the optimized geometry } \\
\hline C3-C4-C5-C6 & -173.8 & -173.5 & -169.2 & -175.5 & -176.1 \\
\hline C4-C5-C6-C7 & -166.5 & -167.6 & -172.2 & -163.2 & -164.9 \\
\hline C8-C9-C10-C11 & 168.7 & 167.1 & 169.1 & 170.2 & 168.6 \\
\hline C9-C10-C11-C12 & 163.4 & 159.6 & 158.7 & 162.6 & 157.2 \\
\hline C13-C14-C15-C16 & 34.0 & 38.1 & 35.9 & 33.2 & 36.4 \\
\hline $\mathrm{C} 14-\mathrm{C} 15-\mathrm{C} 16-\mathrm{C} 17$ & -176.6 & 15.9 & 16.7 & -175.3 & 15.9 \\
\hline \multicolumn{6}{|c|}{ Starting geometries (crystal structures) ${ }^{\mathrm{a}}$} \\
\hline $\mathrm{C} 3-\mathrm{C} 4-\mathrm{C} 5-\mathrm{C} 6$ & -172.4 & -172.5 & -163.4 & -174.7 & -174.7 \\
\hline C4-C5-C6-C7 & -174.2 & -165.5 & -174.3 & 179.9 & 179.9 \\
\hline C8-C9-C10-C11 & 178.6 & 179.9 & -178.0 & -179.7 & -179.7 \\
\hline C9-C10-C11-C12 & -179.2 & -179.8 & -179.7 & 179.9 & 179.9 \\
\hline $\mathrm{C} 13-\mathrm{C} 14-\mathrm{C} 15-\mathrm{C} 16$ & 23.2 & 35.0 & 31.1 & 27.7 & 27.7 \\
\hline C14-C15-C16-C17 & -158.4 & 37.7 & 30.6 & -136.6 & 13.4 \\
\hline
\end{tabular}

a) The Pfr AtPhyB starting structure was produced by modifying the Pr AtPhyB starting structure. 
Table S3. Bond length of tetrapyrroles in different models. (Atom numbering in Figure S3b)

\begin{tabular}{|c|c|c|c|c|c|}
\hline & Pr DrBphP & Pfr DrBphP & Pfr PaBphP & Pr AtPhyB & Pfr AtPhyB \\
\hline \multicolumn{6}{|c|}{ Bond length $(\AA)$} \\
\hline \multicolumn{6}{|c|}{ WT cluster models } \\
\hline S-C & 1.827 & 1.838 & 1.818 & 1.844 & 1.843 \\
\hline C32-C31 & 1.491 & 1.490 & 1.545 & 1.523 & 1.522 \\
\hline C31-C3 & 1.343 & 1.343 & 1.497 & 1.539 & 1.530 \\
\hline $\mathrm{C} 3-\mathrm{C} 4$ & 1.480 & 1.480 & 1.469 & 1.522 & 1.522 \\
\hline C4-C5 & 1.362 & 1.365 & 1.368 & 1.367 & 1.363 \\
\hline C5-C6 & 1.427 & 1.432 & 1.432 & 1.424 & 1.426 \\
\hline C6-C7 & 1.419 & 1.414 & 1.418 & 1.437 & 1.425 \\
\hline $\mathrm{C} 7-\mathrm{C} 8$ & 1.392 & 1.401 & 1.400 & 1.385 & 1.389 \\
\hline C8-C9 & 1.423 & 1.416 & 1.419 & 1.437 & 1.427 \\
\hline C9-C10 & 1.401 & 1.403 & 1.408 & 1.385 & 1.398 \\
\hline C10-C11 & 1.387 & 1.383 & 1.382 & 1.399 & 1.390 \\
\hline C11-C12 & 1.439 & 1.438 & 1.440 & 1.424 & 1.435 \\
\hline C12-C13 & 1.392 & 1.389 & 1.385 & 1.400 & 1.391 \\
\hline C13-C14 & 1.432 & 1.437 & 1.441 & 1.423 & 1.433 \\
\hline C14-C15 & 1.434 & 1.419 & 1.419 & 1.445 & 1.425 \\
\hline C15-C16 & 1.366 & 1.376 & 1.375 & 1.359 & 1.375 \\
\hline C16-C17 & 1.470 & 1.469 & 1.466 & 1.464 & 1.473 \\
\hline C17-C18 & 1.361 & 1.363 & 1.367 & 1.367 & 1.364 \\
\hline C18-C19 & 1.446 & 1.447 & 1.444 & 1.449 & 1.445 \\
\hline C19-C20 & 1.342 & 1.343 & 1.344 & 1.342 & 1.344 \\
\hline C18-C19' & 1.492 & 1.493 & 1.494 & 1.489 & 1.502 \\
\hline C1-NA & 1.370 & 1.369 & 1.384 & 1.385 & 1.382 \\
\hline C4-NA & 1.379 & 1.373 & 1.380 & 1.358 & 1.361 \\
\hline C6-NB & 1.361 & 1.368 & 1.362 & 1.352 & 1.354 \\
\hline C9-NB & 1.385 & 1.386 & 1.379 & 1.388 & 1.383 \\
\hline C11-NC & 1.382 & 1.380 & 1.377 & 1.374 & 1.378 \\
\hline C14-NC & 1.354 & 1.354 & 1.346 & 1.355 & 1.353 \\
\hline C16-ND & 1.373 & 1.370 & 1.368 & 1.386 & 1.366 \\
\hline C19'-ND & 1.385 & 1.373 & 1.384 & 1.379 & 1.380 \\
\hline $\mathrm{C} 1-\mathrm{OA}$ & 1.216 & 1.213 & 1.221 & 1.210 & 1.208 \\
\hline C19'-OD & 1.217 & 1.224 & 1.216 & 1.220 & 1.214 \\
\hline NA-H & 1.043 & 1.038 & 1.036 & 1.039 & 1.034 \\
\hline NB-H & 1.023 & 1.027 & 1.029 & 1.024 & 1.028 \\
\hline NC-H & 1.033 & 1.037 & 1.036 & 1.028 & 1.038 \\
\hline ND-H & 1.034 & 1.067 & 1.031 & 1.007 & 1.048 \\
\hline \multicolumn{6}{|c|}{ Cluster models w/o AspD } \\
\hline S-C & 1.826 & 1.833 & 1.814 & 1.843 & 1.843 \\
\hline C32-C31 & 1.490 & 1.490 & 1.548 & 1.522 & 1.523 \\
\hline C31-C3 & 1.344 & 1.344 & 1.498 & 1.540 & 1.533 \\
\hline $\mathrm{C} 3-\mathrm{C} 4$ & 1.478 & 1.478 & 1.467 & 1.519 & 1.520 \\
\hline $\mathrm{C} 4-\mathrm{C} 5$ & 1.361 & 1.366 & 1.368 & 1.362 & 1.361 \\
\hline C5-C6 & 1.429 & 1.429 & 1.431 & 1.427 & 1.426 \\
\hline
\end{tabular}




\begin{tabular}{|c|c|c|c|c|c|}
\hline C6-C7 & 1.421 & 1.418 & 1.420 & 1.434 & 1.428 \\
\hline C7-C8 & 1.393 & 1.397 & 1.399 & 1.382 & 1.385 \\
\hline C8-C9 & 1.423 & 1.422 & 1.419 & 1.437 & 1.434 \\
\hline C9-C10 & 1.399 & 1.395 & 1.404 & 1.383 & 1.388 \\
\hline C10-C11 & 1.388 & 1.390 & 1.382 & 1.400 & 1.398 \\
\hline C11-C12 & 1.436 & 1.431 & 1.439 & 1.422 & 1.429 \\
\hline C12-C13 & 1.393 & 1.394 & 1.389 & 1.402 & 1.397 \\
\hline C13-C14 & 1.429 & 1.426 & 1.442 & 1.417 & 1.423 \\
\hline C14-C15 & 1.435 & 1.431 & 1.430 & 1.444 & 1.435 \\
\hline C15-C16 & 1.367 & 1.365 & 1.362 & 1.357 & 1.368 \\
\hline C16-C17 & 1.469 & 1.460 & 1.448 & 1.461 & 1.466 \\
\hline C17-C18 & 1.361 & 1.371 & 1.379 & 1.367 & 1.368 \\
\hline C18-C19 & 1.447 & 1.448 & 1.443 & 1.447 & 1.447 \\
\hline C19-C20 & 1.343 & 1.343 & 1.343 & 1.343 & 1.343 \\
\hline C18-C19' & 1.496 & 1.483 & 1.476 & 1.494 & 1.492 \\
\hline C1-NA & 1.362 & 1.367 & 1.379 & 1.374 & 1.377 \\
\hline C4-NA & 1.392 & 1.375 & 1.384 & 1.364 & 1.365 \\
\hline C6-NB & 1.361 & 1.365 & 1.361 & 1.352 & 1.353 \\
\hline C9-NB & 1.385 & 1.387 & 1.377 & 1.389 & 1.386 \\
\hline C11-NC & 1.383 & 1.379 & 1.375 & 1.376 & 1.379 \\
\hline C14-NC & 1.356 & 1.352 & 1.344 & 1.357 & 1.354 \\
\hline C16-ND & 1.372 & 1.391 & 1.406 & 1.383 & 1.378 \\
\hline C19'-ND & 1.383 & 1.379 & 1.408 & 1.377 & 1.386 \\
\hline C1-OA & 1.227 & 1.217 & 1.233 & 1.221 & 1.214 \\
\hline C19'-OD & 1.216 & 1.223 & 1.213 & 1.217 & 1.212 \\
\hline NA-H & 1.046 & 1.039 & 1.040 & 1.042 & 1.030 \\
\hline NB-H & 1.020 & 1.026 & 1.031 & 1.026 & 1.027 \\
\hline NC-H & 1.030 & 1.038 & 1.043 & 1.023 & 1.036 \\
\hline ND-H & 1.041 & 1.006 & 1.004 & 1.012 & 1.008 \\
\hline \multicolumn{6}{|c|}{ Cluster models with HisD(H) } \\
\hline $\mathrm{S}-\mathrm{C}$ & 1.825 & 1.834 & 1.817 & 1.843 & -- \\
\hline C32-C31 & 1.492 & 1.491 & 1.546 & 1.520 & \\
\hline C31-C3 & 1.344 & 1.344 & 1.497 & 1.533 & \\
\hline C3-C4 & 1.480 & 1.479 & 1.473 & 1.520 & \\
\hline C4-C5 & 1.367 & 1.370 & 1.368 & 1.369 & \\
\hline C5-C6 & 1.423 & 1.427 & 1.430 & 1.418 & \\
\hline C6-C7 & 1.428 & 1.421 & 1.421 & 1.438 & \\
\hline $\mathrm{C} 7-\mathrm{C} 8$ & 1.386 & 1.397 & 1.396 & 1.380 & \\
\hline C8-C9 & 1.431 & 1.424 & 1.422 & 1.441 & \\
\hline C9-C10 & 1.391 & 1.395 & 1.401 & 1.377 & \\
\hline $\mathrm{C} 10-\mathrm{C} 11$ & 1.399 & 1.391 & 1.386 & 1.406 & \\
\hline C11-C12 & 1.432 & 1.433 & 1.434 & 1.421 & \\
\hline C12-C13 & 1.397 & 1.394 & 1.389 & 1.405 & \\
\hline C13-C14 & 1.422 & 1.430 & 1.438 & 1.416 & \\
\hline C14-C15 & 1.437 & 1.422 & 1.424 & 1.444 & \\
\hline C15-C16 & 1.362 & 1.369 & 1.372 & 1.358 & \\
\hline C16-C17 & 1.466 & 1.469 & 1.468 & 1.458 & \\
\hline C17-C18 & 1.365 & 1.362 & 1.367 & 1.372 & \\
\hline C18-C19 & 1.448 & 1.446 & 1.443 & 1.450 & \\
\hline
\end{tabular}




\begin{tabular}{|c|c|c|c|c|c|}
\hline C19-C20 & 1.342 & 1.342 & 1.343 & 1.341 & \\
\hline C18-C19' & 1.484 & 1.503 & 1.495 & 1.475 & \\
\hline C1-NA & 1.374 & 1.372 & 1.385 & 1.386 & \\
\hline C4-NA & 1.374 & 1.368 & 1.377 & 1.355 & \\
\hline C6-NB & 1.357 & 1.368 & 1.361 & 1.351 & \\
\hline C9-NB & 1.388 & 1.390 & 1.381 & 1.390 & \\
\hline C11-NC & 1.379 & 1.377 & 1.376 & 1.374 & \\
\hline C14-NC & 1.356 & 1.352 & 1.345 & 1.356 & \\
\hline C16-ND & 1.383 & 1.372 & 1.368 & 1.391 & \\
\hline C19'-ND & 1.373 & 1.370 & 1.382 & 1.362 & \\
\hline C1-OA & 1.215 & 1.213 & 1.220 & 1.210 & \\
\hline C19'-OD & 1.227 & 1.223 & 1.216 & 1.239 & \\
\hline NA-H & 1.047 & 1.036 & 1.033 & 1.036 & \\
\hline NB-H & 1.025 & 1.029 & 1.032 & 1.033 & \\
\hline NC-H & 1.030 & 1.035 & 1.039 & 1.029 & \\
\hline ND-H & 1.027 & 1.069 & 1.034 & 1.012 & \\
\hline \multicolumn{6}{|c|}{ Cluster models w/o HisD } \\
\hline $\mathrm{S}-\mathrm{C}$ & 1.827 & -- & -- & 1.843 & -- \\
\hline C32-C31 & 1.491 & & & 1.523 & \\
\hline C31-C3 & 1.343 & & & 1.539 & \\
\hline $\mathrm{C} 3-\mathrm{C} 4$ & 1.480 & & & 1.520 & \\
\hline $\mathrm{C} 4-\mathrm{C} 5$ & 1.363 & & & 1.366 & \\
\hline C5-C6 & 1.428 & & & 1.425 & \\
\hline C6-C7 & 1.420 & & & 1.433 & \\
\hline $\mathrm{C} 7-\mathrm{C} 8$ & 1.395 & & & 1.383 & \\
\hline C8-C9 & 1.421 & & & 1.435 & \\
\hline C9-C10 & 1.404 & & & 1.387 & \\
\hline $\mathrm{C} 10-\mathrm{C} 11$ & 1.385 & & & 1.397 & \\
\hline C11-C12 & 1.439 & & & 1.424 & \\
\hline $\mathrm{C} 12-\mathrm{C} 13$ & 1.388 & & & 1.398 & \\
\hline C13-C14 & 1.433 & & & 1.422 & \\
\hline $\mathrm{C} 14-\mathrm{C} 15$ & 1.431 & & & 1.440 & \\
\hline $\mathrm{C} 15-\mathrm{C} 16$ & 1.371 & & & 1.360 & \\
\hline $\mathrm{C} 16-\mathrm{C} 17$ & 1.473 & & & 1.463 & \\
\hline $\mathrm{C} 17-\mathrm{C} 18$ & 1.361 & & & 1.366 & \\
\hline C18-C19 & 1.447 & & & 1.449 & \\
\hline C19-C20 & 1.341 & & & 1.342 & \\
\hline C18-C19' & 1.496 & & & 1.493 & \\
\hline C1-NA & 1.369 & & & 1.385 & \\
\hline C4-NA & 1.382 & & & 1.357 & \\
\hline C6-NB & 1.361 & & & 1.352 & \\
\hline C9-NB & 1.382 & & & 1.387 & \\
\hline C11-NC & 1.384 & & & 1.378 & \\
\hline C14-NC & 1.355 & & & 1.356 & \\
\hline C16-ND & 1.368 & & & 1.380 & \\
\hline C19'-ND & 1.389 & & & 1.386 & \\
\hline C1-OA & 1.216 & & & 1.209 & \\
\hline C19'-OD & 1.214 & & & 1.219 & \\
\hline NA-H & 1.044 & & & 1.042 & \\
\hline
\end{tabular}




\begin{tabular}{|c|c|c|c|c|c|}
\hline NB-H & 1.022 & & & 1.024 & \\
\hline NC-H & 1.032 & & & 1.026 & \\
\hline ND-H & 1.044 & & & 1.009 & \\
\hline \multicolumn{6}{|c|}{ Tetrapyrrole chromophores at the optimized geometry } \\
\hline S-C & 1.830 & 1.831 & 1.811 & 1.835 & 1.835 \\
\hline C32-C31 & 1.486 & 1.486 & 1.535 & 1.521 & 1.521 \\
\hline C31-C3 & 1.346 & 1.346 & 1.491 & 1.538 & 1.539 \\
\hline C3-C4 & 1.466 & 1.466 & 1.464 & 1.516 & 1.517 \\
\hline C4-C5 & 1.372 & 1.373 & 1.366 & 1.363 & 1.363 \\
\hline C5-C6 & 1.420 & 1.421 & 1.429 & 1.427 & 1.427 \\
\hline C6-C7 & 1.417 & 1.418 & 1.414 & 1.414 & 1.415 \\
\hline C7-C8 & 1.399 & 1.399 & 1.405 & 1.400 & 1.401 \\
\hline C8-C9 & 1.421 & 1.420 & 1.418 & 1.420 & 1.420 \\
\hline C9-C10 & 1.391 & 1.393 & 1.403 & 1.392 & 1.395 \\
\hline C10-C11 & 1.397 & 1.398 & 1.391 & 1.396 & 1.397 \\
\hline C11-C12 & 1.417 & 1.417 & 1.424 & 1.417 & 1.417 \\
\hline C12-C13 & 1.404 & 1.404 & 1.399 & 1.403 & 1.404 \\
\hline C13-C14 & 1.417 & 1.417 & 1.424 & 1.418 & 1.418 \\
\hline C14-C15 & 1.430 & 1.429 & 1.423 & 1.430 & 1.427 \\
\hline C15-C16 & 1.367 & 1.371 & 1.373 & 1.368 & 1.371 \\
\hline C16-C17 & 1.466 & 1.467 & 1.465 & 1.466 & 1.466 \\
\hline C17-C18 & 1.365 & 1.367 & 1.367 & 1.366 & 1.367 \\
\hline C18-C19 & 1.446 & 1.444 & 1.443 & 1.446 & 1.444 \\
\hline C19-C20 & 1.343 & 1.344 & 1.345 & 1.343 & 1.344 \\
\hline C18-C19' & 1.497 & 1.497 & 1.500 & 1.497 & 1.498 \\
\hline C1-NA & 1.384 & 1.385 & 1.403 & 1.386 & 1.385 \\
\hline C4-NA & 1.380 & 1.382 & 1.394 & 1.370 & 1.373 \\
\hline C6-NB & 1.375 & 1.373 & 1.367 & 1.373 & 1.369 \\
\hline C9-NB & 1.397 & 1.397 & 1.387 & 1.397 & 1.396 \\
\hline C11-NC & 1.393 & 1.393 & 1.390 & 1.394 & 1.393 \\
\hline C14-NC & 1.371 & 1.372 & 1.367 & 1.372 & 1.372 \\
\hline C16-ND & 1.371 & 1.375 & 1.372 & 1.371 & 1.374 \\
\hline C19'-ND & 1.398 & 1.389 & 1.390 & 1.399 & 1.388 \\
\hline C1-OA & 1.209 & 1.210 & 1.217 & 1.209 & 1.211 \\
\hline C19'-OD & 1.208 & 1.209 & 1.208 & 1.208 & 1.209 \\
\hline NA-H & 1.011 & 1.011 & 1.013 & 1.011 & 1.011 \\
\hline NB-H & 1.016 & 1.011 & 1.015 & 1.016 & 1.015 \\
\hline NC-H & 1.013 & 1.014 & 1.012 & 1.013 & 1.014 \\
\hline ND-H & 1.010 & 1.013 & 1.015 & 1.010 & 1.014 \\
\hline
\end{tabular}


Table S4. Charge analysis. Atomic and net Mulliken charges in the $\mathrm{S}_{0}$ state and $\mathrm{S}_{1}$ states computed for the WT cluster models. Fragment ABC includes rings A, B, C and methine bridges AB, BC. Fragment D includes ring $\mathrm{D}$ and methine bridge $\mathrm{CD}$. Fragment $\mathrm{A}$ includes ring $\mathrm{A}$ and methine bridge $\mathrm{AB}$. Fragment $\mathrm{BCD}$ includes rings $\mathrm{B}, \mathrm{C}, \mathrm{D}$ and methine bridges $\mathrm{BC}, \mathrm{CD}$. "br" indicates methine bridge between two rings.

\begin{tabular}{|c|c|c|c|c|c|}
\hline & Pr DrBphP & Pfr DrBphP & Pfr PaBphP & Pr AtPhyB & Pfr AtPhyB \\
\hline \multicolumn{6}{|c|}{$S_{0}$ state PBE0 method } \\
\hline ring $\mathrm{A}$ & 0.055 & 0.131 & 0.011 & 0.168 & 0.176 \\
\hline br $(\mathrm{AB})$ & -0.073 & -0.100 & -0.075 & -0.067 & -0.092 \\
\hline $\operatorname{ring} \mathrm{B}$ & 0.192 & 0.178 & 0.206 & 0.254 & 0.191 \\
\hline br (BC) & -0.004 & -0.047 & -0.038 & 0.014 & -0.032 \\
\hline ring $\mathrm{C}$ & 0.239 & 0.233 & 0.296 & 0.215 & 0.211 \\
\hline br (CD) & -0.085 & -0.023 & -0.064 & -0.037 & -0.016 \\
\hline $\operatorname{ring} \mathrm{D}$ & 0.077 & 0.086 & 0.130 & 0.089 & 0.128 \\
\hline Total & 0.400 & 0.459 & 0.466 & 0.636 & 0.566 \\
\hline atom $\mathrm{N}(\mathrm{A})$ & -0.211 & -0.182 & -0.239 & -0.171 & -0.187 \\
\hline atom $\mathrm{N}(\mathrm{B})$ & -0.213 & -0.244 & -0.232 & -0.196 & -0.202 \\
\hline atom N (C) & -0.201 & -0.210 & -0.200 & -0.189 & -0.203 \\
\hline atom $\mathrm{N}(\mathrm{D})$ & -0.224 & -0.204 & -0.193 & -0.197 & -0.204 \\
\hline Frag ABC & 0.409 & 0.396 & 0.400 & 0.584 & 0.455 \\
\hline Frag D & -0.008 & 0.063 & 0.066 & 0.052 & 0.111 \\
\hline Frag A & -0.019 & 0.031 & -0.064 & 0.101 & 0.084 \\
\hline Frag BCD & 0.419 & 0.427 & 0.530 & 0.535 & 0.482 \\
\hline \multicolumn{6}{|c|}{$S_{0}$ state XMCQDPT2 method } \\
\hline Ring A & 0.118 & 0.197 & 0.065 & 0.226 & 0.223 \\
\hline br (AB) & -0.109 & -0.143 & -0.087 & -0.108 & -0.127 \\
\hline ring $\mathrm{B}$ & 0.189 & 0.188 & 0.190 & 0.295 & 0.216 \\
\hline br (BC) & 0.104 & 0.054 & 0.047 & 0.119 & 0.067 \\
\hline $\operatorname{ring} \mathrm{C}$ & 0.303 & 0.282 & 0.360 & 0.156 & 0.249 \\
\hline br (CD) & -0.092 & -0.018 & -0.070 & -0.037 & -0.024 \\
\hline ring D & 0.094 & 0.101 & 0.140 & 0.150 & 0.186 \\
\hline Total & 0.607 & 0.660 & 0.645 & 0.801 & 0.789 \\
\hline atom N (A) & -0.401 & -0.372 & -0.420 & -0.367 & -0.378 \\
\hline atom N (B) & -0.373 & -0.396 & -0.380 & -0.365 & -0.363 \\
\hline atom $\mathrm{N}(\mathrm{C})$ & -0.347 & -0.356 & -0.347 & -0.343 & -0.349 \\
\hline atom $\mathrm{N}(\mathrm{D})$ & -0.434 & -0.418 & -0.392 & -0.367 & -0.403 \\
\hline Frag. ABC & 0.606 & 0.577 & 0.575 & 0.688 & 0.627 \\
\hline Frag. D & 0.002 & 0.083 & 0.070 & 0.113 & 0.162 \\
\hline Frag. A & 0.009 & 0.053 & -0.022 & 0.118 & 0.096 \\
\hline Frag. BCD & 0.598 & 0.607 & 0.667 & 0.682 & 0.693 \\
\hline \multicolumn{6}{|c|}{$S_{1}$ state XMCQDPT2 method } \\
\hline Ring A & 0.128 & 0.211 & 0.078 & 0.173 & 0.220 \\
\hline br $(\mathrm{AB})$ & -0.090 & -0.125 & -0.083 & -0.084 & -0.107 \\
\hline ring $\mathrm{B}$ & 0.314 & 0.301 & 0.334 & 0.234 & 0.279 \\
\hline br (BC) & -0.077 & -0.095 & -0.089 & 0.026 & -0.103 \\
\hline $\operatorname{ring} \mathrm{C}$ & 0.327 & 0.258 & 0.315 & 0.315 & 0.274 \\
\hline
\end{tabular}




\begin{tabular}{|c|c|c|c|c|c|}
\hline br (CD) & -0.072 & 0.019 & -0.030 & -0.033 & 0.019 \\
\hline ring D & 0.077 & 0.092 & 0.121 & 0.171 & 0.206 \\
\hline Total & 0.606 & 0.661 & 0.646 & 0.802 & 0.789 \\
\hline
\end{tabular}

Table S5. Charge analysis. Atomic and net Mulliken charges in the $S_{0}$ state and $S_{1}$ states computed for the cluster models w/o AspD, and with protonated $\operatorname{HisD}(\mathrm{H})$. Fragment $\mathrm{ABC}$ includes rings $\mathrm{A}, \mathrm{B}, \mathrm{C}$ and methine bridges $\mathrm{AB}, \mathrm{BC}$. Fragment $\mathrm{D}$ includes ring $\mathrm{D}$ and methine bridge $\mathrm{CD}$. Fragment $\mathrm{A}$ includes ring $\mathrm{A}$ and methine bridge $\mathrm{AB}$. Fragment $\mathrm{BCD}$ includes rings $\mathrm{B}, \mathrm{C}, \mathrm{D}$ and methine bridges $\mathrm{BC}, \mathrm{CD}$. "br" indicates methine bridge between two rings.

\begin{tabular}{|c|c|c|c|c|c|}
\hline & Pr DrBphP & Pfr DrBphP & Pfr PaBphP & Pr AtPhyB & Pfr AtPhyB \\
\hline \multicolumn{6}{|c|}{ Cluster models w/o AspD } \\
\hline \multicolumn{6}{|c|}{$\mathrm{S}_{0}$ state $\mathrm{PBE} 0$ method } \\
\hline ring $\mathrm{A}$ & -0.003 & 0.119 & -0.051 & 0.141 & 0.182 \\
\hline br $(\mathrm{AB})$ & -0.049 & -0.084 & -0.040 & -0.050 & -0.070 \\
\hline ring $\mathrm{B}$ & 0.210 & 0.232 & 0.254 & 0.265 & 0.242 \\
\hline br (BC) & 0.011 & -0.017 & -0.004 & 0.028 & -0.008 \\
\hline $\operatorname{ring} \mathrm{C}$ & 0.254 & 0.236 & 0.312 & 0.222 & 0.213 \\
\hline br (CD) & -0.107 & -0.037 & -0.063 & -0.055 & -0.045 \\
\hline $\operatorname{ring} \mathrm{D}$ & 0.082 & 0.096 & 0.144 & 0.091 & 0.136 \\
\hline Total & 0.397 & 0.545 & 0.552 & 0.642 & 0.649 \\
\hline atom $\mathrm{N}(\mathrm{A})$ & -0.207 & -0.184 & -0.240 & -0.172 & -0.179 \\
\hline atom $\mathrm{N}(\mathrm{B})$ & -0.212 & -0.241 & -0.232 & -0.206 & -0.208 \\
\hline atom $\mathrm{N}(\mathrm{C})$ & -0.204 & -0.209 & -0.204 & -0.190 & -0.202 \\
\hline atom $\mathrm{N}(\mathrm{D})$ & -0.228 & -0.173 & -0.246 & -0.198 & -0.190 \\
\hline Frag ABC & 0.423 & 0.486 & 0.471 & 0.607 & 0.559 \\
\hline Frag D & -0.026 & 0.059 & 0.081 & 0.036 & 0.090 \\
\hline \multicolumn{6}{|c|}{$\mathrm{S}_{0}$ state XMCQDPT2 method } \\
\hline ring $\mathrm{A}$ & 0.057 & 0.180 & -0.019 & 0.201 & 0.229 \\
\hline br $(\mathrm{AB})$ & -0.076 & -0.128 & -0.043 & -0.080 & -0.107 \\
\hline ring $\mathrm{B}$ & 0.234 & 0.244 & 0.210 & 0.330 & 0.267 \\
\hline br (BC) & 0.142 & 0.085 & 0.091 & 0.122 & 0.098 \\
\hline $\operatorname{ring} \mathrm{C}$ & 0.289 & 0.264 & 0.335 & 0.115 & 0.172 \\
\hline br (CD) & -0.099 & 0.005 & -0.047 & -0.008 & -0.033 \\
\hline ring D & 0.077 & 0.060 & 0.161 & 0.117 & 0.193 \\
\hline Total & 0.624 & 0.711 & 0.687 & 0.796 & 0.818 \\
\hline atom $\mathrm{N}(\mathrm{A})$ & -0.396 & -0.373 & -0.424 & -0.366 & -0.370 \\
\hline atom $\mathrm{N}(\mathrm{B})$ & -0.364 & -0.394 & -0.380 & -0.375 & -0.370 \\
\hline atom $\mathrm{N}(\mathrm{C})$ & -0.344 & -0.351 & -0.356 & -0.360 & -0.351 \\
\hline atom N (D) & -0.420 & -0.347 & -0.394 & -0.373 & -0.348 \\
\hline Frag $\mathrm{ABC}$ & 0.646 & 0.646 & 0.574 & 0.687 & 0.659 \\
\hline Frag D & -0.022 & 0.065 & 0.114 & 0.108 & 0.160 \\
\hline \multicolumn{6}{|c|}{$\mathrm{S}_{1}$ state XMCQDPT2 method } \\
\hline Ring A & 0.074 & 0.181 & -0.020 & 0.169 & 0.191 \\
\hline br $(\mathrm{AB})$ & -0.058 & -0.103 & -0.037 & -0.057 & -0.085 \\
\hline ring $\mathrm{B}$ & 0.353 & 0.331 & 0.384 & 0.306 & 0.257 \\
\hline
\end{tabular}




\begin{tabular}{|c|c|c|c|c|c|}
\hline br (BC) & -0.060 & -0.104 & 0.012 & -0.064 & -0.003 \\
\hline ring $\mathrm{C}$ & 0.342 & 0.334 & 0.284 & 0.316 & 0.296 \\
\hline br (CD) & -0.089 & -0.005 & -0.034 & -0.009 & -0.032 \\
\hline ring D & 0.058 & 0.076 & 0.099 & 0.131 & 0.196 \\
\hline Total & 0.619 & 0.711 & 0.688 & 0.793 & 0.820 \\
\hline \multicolumn{6}{|c|}{ Cluster models with protonated HisD(H) } \\
\hline \multicolumn{6}{|c|}{$\mathrm{S}_{0}$ state $\mathrm{PBE} 0$ method } \\
\hline ring $\mathrm{A}$ & 0.103 & 0.171 & 0.048 & 0.219 & -- \\
\hline br $(\mathrm{AB})$ & -0.073 & -0.098 & -0.070 & -0.062 & \\
\hline ring $\mathrm{B}$ & 0.243 & 0.215 & 0.234 & 0.284 & \\
\hline br (BC) & 0.006 & -0.045 & -0.037 & -0.002 & \\
\hline $\operatorname{ring} \mathrm{C}$ & 0.194 & 0.222 & 0.279 & 0.200 & \\
\hline br (CD) & -0.055 & -0.035 & -0.055 & 0.006 & \\
\hline ring D & 0.015 & 0.025 & 0.091 & 0.044 & \\
\hline Total & 0.431 & 0.456 & 0.490 & 0.689 & \\
\hline atom $\mathrm{N}(\mathrm{A})$ & -0.216 & -0.180 & -0.232 & -0.185 & \\
\hline atom $\mathrm{N}(\mathrm{B})$ & -0.221 & -0.252 & -0.237 & -0.218 & \\
\hline atom $\mathrm{N}(\mathrm{C})$ & -0.194 & -0.196 & -0.195 & -0.189 & \\
\hline atom $\mathrm{N}(\mathrm{D})$ & -0.218 & -0.205 & -0.189 & -0.184 & \\
\hline Frag ABC & 0.471 & 0.466 & 0.454 & 0.639 & \\
\hline Frag D & -0.040 & -0.009 & 0.036 & 0.050 & \\
\hline Frag A & 0.029 & 0.073 & -0.022 & 0.157 & \\
\hline Frag BCD & 0.402 & 0.383 & 0.512 & 0.532 & \\
\hline \multicolumn{6}{|c|}{$\mathrm{S}_{0}$ state XMCQDPT2 } \\
\hline ring $\mathrm{A}$ & 0.169 & 0.234 & 0.099 & 0.284 & \\
\hline br $(\mathrm{AB})$ & -0.118 & -0.148 & -0.089 & -0.109 & \\
\hline ring $\mathrm{B}$ & 0.255 & 0.248 & 0.235 & 0.339 & \\
\hline br (BC) & 0.108 & 0.057 & 0.051 & 0.092 & \\
\hline $\operatorname{ring} \mathrm{C}$ & 0.227 & 0.248 & 0.327 & 0.108 & \\
\hline br (CD) & -0.038 & -0.021 & -0.059 & 0.017 & \\
\hline ring $\mathrm{D}$ & 0.022 & 0.041 & 0.104 & 0.114 & \\
\hline Total & 0.626 & 0.660 & 0.667 & 0.844 & \\
\hline atom $\mathrm{N}(\mathrm{A})$ & -0.408 & -0.369 & -0.413 & -0.378 & \\
\hline atom $\mathrm{N}(\mathrm{B})$ & -0.381 & -0.407 & -0.387 & -0.384 & \\
\hline atom $\mathrm{N}(\mathrm{C})$ & -0.338 & -0.342 & -0.341 & -0.337 & \\
\hline atom $\mathrm{N}(\mathrm{D})$ & -0.419 & -0.418 & -0.393 & -0.353 & \\
\hline Frag $\mathrm{ABC}$ & 0.641 & 0.640 & 0.622 & 0.714 & \\
\hline Frag D & -0.015 & 0.020 & 0.045 & 0.131 & \\
\hline Frag A & 0.050 & 0.086 & 0.009 & 0.175 & \\
\hline Frag BCD & 0.575 & 0.574 & 0.658 & 0.670 & \\
\hline \multicolumn{6}{|c|}{$\mathrm{S}_{1}$ state XMCQDPT2 method } \\
\hline Ring A & 0.149 & 0.231 & 0.102 & 0.212 & \\
\hline br (AB) & -0.089 & -0.123 & -0.079 & -0.084 & \\
\hline ring $\mathrm{B}$ & 0.310 & 0.304 & 0.350 & 0.218 & \\
\hline br (BC) & -0.083 & -0.116 & -0.110 & 0.024 & \\
\hline ring $\mathrm{C}$ & 0.332 & 0.280 & 0.320 & 0.363 & \\
\hline br (CD) & -0.049 & 0.007 & -0.026 & 0.004 & \\
\hline ring D & 0.055 & 0.077 & 0.113 & 0.110 & \\
\hline Total & 0.624 & 0.659 & 0.669 & 0.847 & \\
\hline
\end{tabular}


Table S6. Main characteristics of the cluster models. The standard one-letter residue notation is used. "PW" and "W" indicate pyrrole water and water, respectively.

\begin{tabular}{|c|c|c|c|c|c|}
\hline & Pr DrBphP & Pfr DrBphP & Pfr PaBphP & Pr AtPhyB & Pfr AtPhyB \\
\hline Parent pdb & $209 c^{9} *$ & $5 \mathrm{c} 5 \mathrm{k}^{12}$ & $3 \mathrm{c} 2 \mathrm{w}^{10}$ & 4 our $^{13}$ & 4 our $^{13}$ \\
\hline $\begin{array}{l}\text { Model } \\
\text { composition }\end{array}$ & $\begin{array}{l}\text { BV, C24, } \\
\text { D207, Y216, } \\
\text { R254, H260, } \\
\text { S272, S274, } \\
\text { Y263, H290, } \\
\text { R466, PW, } \\
\text { W330, W331 }\end{array}$ & $\begin{array}{l}\text { BV, C24, } \\
\text { Y176, H201, } \\
\text { D207, R222, } \\
\text { H260, S272, } \\
\text { Y263, H290, } \\
\text { S468, PW }\end{array}$ & $\begin{array}{l}\text { BV, C12, } \\
\text { Y163, D194, } \\
\text { R209, H247, } \\
\text { Y250, S259, } \\
\text { S275, H277, } \\
\text { R453, S459, } \\
\text { PW, W905 }\end{array}$ & $\begin{array}{l}\text { PФB, C357, } \\
\text { D307, R322, } \\
\text { R352, H358, } \\
\text { Y361, H403, } \\
\text { R582, PW, W } \\
\text { (between } \\
\text { PropC and } \\
\text { H358) }\end{array}$ & $\begin{array}{l}\text { C357, } \\
\text { D307, } \\
\text { R322, } \\
\text { R352, H358 } \\
\text { Y361, PW, } \\
\text { W(between } \\
\text { PropC and } \\
\text { H358) }\end{array}$ \\
\hline $\begin{array}{l}\text { Charges of } \\
\text { the molecular } \\
\text { fragments } \\
\text { depending on } \\
\text { protonation } \\
\text { states }\end{array}$ & $\begin{array}{l}\text { BV }(+1), \\
\text { PropB }(-1), \\
\text { PropC }(-1), \\
\text { D207(-1), } \\
\text { R254(+1), } \\
\text { R466(+1), } \\
\varepsilon H 260(0), \\
\varepsilon H 290(0) / \\
\delta H 290(0) / \\
\mathrm{H} 290(\mathrm{H})(+1)\end{array}$ & 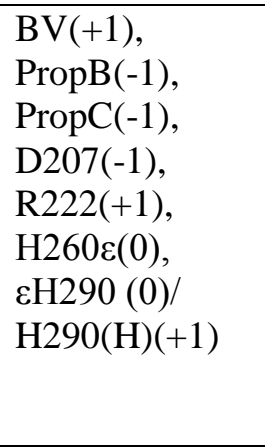 & $\begin{array}{l}\text { BV(+1), } \\
\text { PropB(-1), } \\
\text { PropC(-1), } \\
\text { D194(-1), } \\
\text { R209(+1), } \\
\text { R453(+1), } \\
\varepsilon H 247(0), \\
\varepsilon H 277(0) / \\
\text { H277(H)(+1) }\end{array}$ & 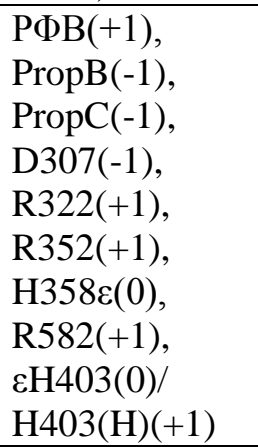 & $\begin{array}{l}\text { PФB(+1), } \\
\text { PropB(-1), } \\
\text { PropC(-1), } \\
\text { D307(-1), } \\
\text { R322(+1), } \\
\text { R352(+1), } \\
\text { عH358 (0), } \\
\text { R582(+1) }\end{array}$ \\
\hline Net charge & $\begin{array}{l}0(\varepsilon \mathrm{H} 290) \\
0(\delta \mathrm{H} 290) \\
+1(\mathrm{H} 290(\mathrm{H}))\end{array}$ & $\begin{array}{l}-1(\varepsilon \mathrm{H} 290), \\
0(\mathrm{H} 290(\mathrm{H}))\end{array}$ & $\begin{array}{l}0(\varepsilon \mathrm{H} 277) \\
+1(\mathrm{H} 277(\mathrm{H}))\end{array}$ & $\begin{array}{l}+1(\varepsilon \mathrm{H} 403), \\
+2(\mathrm{H} 403(\mathrm{H}))\end{array}$ & 0 \\
\hline \# atoms & $\begin{array}{l}214(\varepsilon \mathrm{H} 290), \\
214(\delta \mathrm{H} 290), \\
215(\mathrm{H} 290(\mathrm{H}))\end{array}$ & $\begin{array}{l}\text { 201( }(\mathrm{H} 290), \\
202(\mathrm{H} 290(\mathrm{H}))\end{array}$ & $\begin{array}{l}220(\varepsilon H 290), \\
221(\mathrm{HH} 290)\end{array}$ & $\begin{array}{l}\text { 195(عH290), } \\
196(\mathrm{HH} 290)\end{array}$ & 167 \\
\hline $\begin{array}{l}\text { \# basis } \\
\text { functions }\end{array}$ & $\begin{array}{l}\text { 2114(عH290), } \\
2114(\delta \mathrm{H} 290), \\
2119(\mathrm{H} 290(\mathrm{H})\end{array}$ & $\begin{array}{l}\text { 2019(घH290), } \\
2024(\mathrm{H} 290(\mathrm{H}))\end{array}$ & $\begin{array}{l}2164(\mathrm{cH} 290), \\
2165(\mathrm{H} 290(\mathrm{H})\end{array}$ & $\begin{array}{l}\text { 1929(عН290), } \\
1934(\mathrm{H} 290(\mathrm{H}))\end{array}$ & 1669 \\
\hline
\end{tabular}

* $\mathrm{R} 466$ residue was added from the pdb 4o0p ${ }^{11}$ after overlaying the $2 \mathrm{o} 9 \mathrm{c}$ and 4o0p pdb structures ${ }^{9,11}$. 
Table S7. Energy gradients (a.u.) at the last step of geometry optimization.

\begin{tabular}{|c|c|c|c|c|c|}
\hline & Pr DrBphP & Pfr DrBphP & Pfr PaBphP & Pr AtPhyB & Pfr AtPhyB \\
\hline \multicolumn{6}{|c|}{ WT cluster models (containing $\varepsilon H i s D$ and AspD) } \\
\hline Maximum grad & 0.001855 & 0.002022 & 0.00232 & 0.004279 & 0.003063 \\
\hline RMS grad & 0.000391 & 0.000437 & 0.000488 & 0.001024 & 0.000543 \\
\hline RMSD coord. $(\AA)$ & 0.402 & 0.547 & 0.485 & 0.578 & 0.547 \\
\hline \multicolumn{6}{|c|}{ Cluster models w/o AspD (containing $\varepsilon$ HisD) } \\
\hline Maximum grad & 0.006980 & 0.003816 & 0.016246 & 0.022631 & 0.003169 \\
\hline RMS grad & 0.000723 & 0.000568 & 0.002166 & 0.002414 & 0.000480 \\
\hline \multicolumn{6}{|c|}{ Protonated HisD: WT cluster models (containing HisD(H) and AspD) } \\
\hline Maximum grad & 0.003291 & 0.001947 & 0.002769 & 0.003095 & $\mathrm{n} / \mathrm{c}$ \\
\hline RMS grad & 0.000516 & 0.000438 & 0.000511 & 0.000599 & \\
\hline \multicolumn{6}{|c|}{ Neutral $\delta$ HisD: WT cluster models (containing $\delta$ HisD and AspD) } \\
\hline Maximum grad & 0.002651 & $\mathrm{n} / \mathrm{c}$ & $\mathrm{n} / \mathrm{c}$ & $\mathrm{n} / \mathrm{c}$ & $\mathrm{n} / \mathrm{c}$ \\
\hline RMS grad & 0.000498 & & & & \\
\hline \multicolumn{6}{|c|}{ Cluster models w/o HisD (containing AspD) } \\
\hline Maximum grad & 0.002214 & $\mathrm{n} / \mathrm{c}$ & $\mathrm{n} / \mathrm{c}$ & 0.007513 & $\mathrm{n} / \mathrm{c}$ \\
\hline RMS grad & 0.000395 & & & 0.000773 & \\
\hline
\end{tabular}

n/c - not computed;

RMSD is computed between the coordinates of the starting geometry (i.e., pdb coordinates) and geometry at the last step of optimization.

Table S8. List of MD simulations.

\begin{tabular}{|l|l|l|l|c|}
\hline Model & $\begin{array}{l}\text { Protonation } \\
\text { of HisD }\end{array}$ & $\begin{array}{l}\text { Domain } \\
\text { composition }\end{array}$ & $\begin{array}{l}\text { Parent pdb } \\
\text { structure }\end{array}$ & $\begin{array}{l}\text { Computed } \\
\text { trajectory, ns }\end{array}$ \\
\hline PrDr-CBDPHY & $\varepsilon$ & CBD-PHY & $4 \mathrm{o}^{11}$ & 80 \\
\hline PrDr-CBDPHY & $\delta$ & CBD-PHY & $4 \mathrm{o}^{11}$ & 80 \\
\hline PrDr-CBDPHY & $\mathrm{H}$ & CBD-PHY & $4 \mathrm{o}^{11} \mathrm{p}^{11}$ & 80 \\
\hline PfrDr-CBDPHY & $\varepsilon$ & CBD-PHY & $5 \mathrm{c} 5 \mathrm{k}^{12}$ & 80 \\
\hline PfrDr-CBDPHY* & $\mathrm{H}$ & CBD-PHY & $5 \mathrm{c} 5 \mathrm{k}^{12}$ & 80 \\
\hline PfrPa-CBDPHY & $\varepsilon$ & CBD-PHY & $3 \mathrm{c} 2 \mathrm{w}^{10}$ & 80 \\
\hline PfrPa-CBDPHY & $\mathrm{H}$ & CBD-PHY & $3 \mathrm{c} 2 \mathrm{w}^{10}$ & 80 \\
\hline PrDr-CBD & $\mathrm{H}$ & CBD & $2 \mathrm{o} 9 \mathrm{c}^{9}$ & 80 \\
\hline PfrDr-CBD & $\mathrm{H}$ & CBD & $5 \mathrm{c} 5 \mathrm{k}^{12}$ & 80 \\
\hline PfrPa-CBD & $\mathrm{H}$ & CBD & $3 \mathrm{c} 2 \mathrm{w}^{10}$ & 80 \\
\hline Dr-15E-CBD(Pr)PHY(Pr) & $\mathrm{H}$ & CBD-PHY & & 80 \\
\hline Dr-15E-CBD(Pr) & $\mathrm{H}$ & CBD & & 80 \\
\hline Dr-15E-CBD(Pr)PHY(Pfr) & $\mathrm{H}$ & CBD-PHY & & 80 \\
\hline Dr-15E-CBD(Pfr)PHY(Pfr)* & $\mathrm{H}$ & CBD-PHY & $5 \mathrm{c} 5 \mathrm{k}^{12}$ & \\
\hline
\end{tabular}

* Note that PfrDr-CBDPHY and Dr-15E-CBD(Pfr)PHY(Pfr) are the same model. 
Table S9. Force field parameters for 15Ea biliverdin and biliverdin-cysteine attachment in PaBphP. Atom names and types are adapted from Kaminski et $\mathrm{al}^{38}$. Atom names for the 15Ea-biliverdin in Pfr PaBphP are indicated in Figure S10.

\begin{tabular}{|c|c|c|c|}
\hline \multicolumn{4}{|c|}{ Parameters for 15Ea-biliverdin in Pfr DrBphP and Pfr PaBphP models } \\
\hline \multicolumn{4}{|c|}{ Dihedral angle } \\
\hline Atom types & $\begin{array}{c}\text { Dihedral angle, } \\
\text { degree }\end{array}$ & Multiplicity & $\begin{array}{l}\text { Force constant, } \\
\mathrm{kcal} / \mathrm{mol}\end{array}$ \\
\hline $\begin{array}{c}\text { CPA CPY2 CA } \\
\text { CPY5 }\end{array}$ & 180.00 & 1 & 11.80 \\
\hline
\end{tabular}

\section{Parameters for ring A of 15Ea-biliverdin in Pfr PaBphP}

Atomic Charges

\begin{tabular}{|c|c|c|l|}
\hline Atom name & Atom type & Charge, a. u. & \\
\hline CAC & CT2 & 0.018 & \\
\hline HAC & HA & 0.090 & \\
\hline HBC & HA & 0.090 & \\
\hline C2C & CA & 0.215 & \\
\hline
\end{tabular}

Bonds

\begin{tabular}{|c|c|c|c|}
\hline Atom types & Distance, $\AA$ & $\begin{array}{c}\text { Force constant, } \\
\mathrm{kcal} / \mathrm{mol} / \AA^{\wedge} 2\end{array}$ & \\
\hline CT2 CPY1 & 1.4900 & 230.00 & \\
\hline CPM C & 1.4711 & 250.00 & \\
\hline CPM CT3 & 1.4850 & 230.00 & \\
\hline
\end{tabular}

Angles

\begin{tabular}{|c|c|c|c|}
\hline Atom types & Angle, degree & $\begin{array}{c}\text { Force constant, } \\
\mathrm{kcal} / \mathrm{mol}_{\mathrm{rad}} 2\end{array}$ & \\
\hline HA CT2 CPY1 & 109.50 & 50.00 & \\
\hline CT2 CT2 CPY1 & 113.00 & 70.00 & \\
\hline CT2 CPY1 CA & 124.10 & 61.60 & \\
\hline CT2 CPY1 CPM & 126.74 & 70.00 & \\
\hline CA CPY1 CPM & 116.00 & 52.00 & \\
\hline CPY1 CPM C & 116.50 & 40.00 & \\
\hline CPY1 CPM CT3 & 122.30 & 45.80 & \\
\hline CT2 CT2 SE & 114.50 & 58.00 & \\
\hline CT3 CPM C & 115.90 & 45.80 & \\
\hline CPM C O & 121.00 & 80.00 & \\
\hline CPM C NR1 & 109.50 & 20.00 & \\
\hline CPM CT3 HA * & 33.43 & 110.10 & \\
\hline * Urey-Bradley parameters: constant $22.53 \mathrm{kcal} / \mathrm{mol} / \AA$ ^2, Urey-Bradley $2.1790 \AA$ \\
\hline Dihedral angles
\end{tabular}




\begin{tabular}{|c|c|c|c|}
\hline Atom types & Angle, degree & Multiplicity & $\begin{array}{c}\text { Force constant, } \\
\mathrm{kcal} / \mathrm{mol}\end{array}$ \\
\hline HA CT2 CPY1 CA & 0.00 & 3 & 0.00 \\
\hline HA CT2 CPY1 CPM & 0.00 & 3 & 0.00 \\
\hline CT2 CT2 CPY1 CA & 0.00 & 3 & 0.00 \\
\hline CT2 CT2 CPY1 CPM & 0.00 & 3 & 0.00 \\
\hline SE CT2 CT2 CPY1 & 45.00 & 2 & 1.40 \\
\hline HA CT2 CT2 CPY1 & 0.00 & 3 & 0.00 \\
\hline CT2 CPY1 CA CPY3 & 180.00 & 2 & 3.10 \\
\hline CT2 CPY1 CA NR1 & 180.00 & 2 & 3.00 \\
\hline CT2 CPY1 CPM C & 0.00 & 3 & 3.00 \\
\hline CT2 CPY1 CPM CT3 & 180.00 & 2 & 3.10 \\
\hline CA CPY1 CA CPY3 & 180.00 & 2 & 3.10 \\
\hline CA CPY1 CA NR1 & 0.00 & 3 & 0.19 \\
\hline C CA CPY $1 \mathrm{CA}$ & 0.00 & 3 & 0.04 \\
\hline CT3 CA CPY1 CA & 180.00 & 2 & 3.10 \\
\hline CPY1 CPM C NR1 & 0.00 & 1 & 0.00 \\
\hline CPY1 CPM C O & 180.00 & 1 & 1.40 \\
\hline CT2 SE CT2 CT2 & 10.00 & 3 & 0.70 \\
\hline CT2 SE CT2 CT2 & 88.00 & 1 & 1.10 \\
\hline C CPM CPY1 CA & 0.00 & 3 & 0.04 \\
\hline CA CPY1 CPM CT3 & 180.00 & 2 & 3.10 \\
\hline CPY1 CPM CT3 HA & 0.00 & 3 & 0.20 \\
\hline CT3 CPM C NR1 & 0.00 & 1 & 0.00 \\
\hline CT3 CPM C O & 180.00 & 2 & 3.10 \\
\hline HA СТ3 CPM C & 0.00 & 3 & 0.20 \\
\hline CPM C NR1 H & 180.00 & 2 & 2.50 \\
\hline CPM C NR1 CA & 180.00 & 2 & 2.75 \\
\hline \multicolumn{4}{|l|}{ Impropers } \\
\hline Atom types & $\begin{array}{c}\text { Dihedral angle, } \\
\text { degree }\end{array}$ & $\begin{array}{l}\text { Force constant, } \\
\mathrm{kcal} / \mathrm{mol} / \mathrm{rad}^{\wedge} 2\end{array}$ & \\
\hline CPY1 CPM CA CT2 & 0 & 0 & \\
\hline CPY1 CT2 CT2 HA & 180 & 29.4 & \\
\hline
\end{tabular}




\section{S4. Supplementary references}

17. Gaussian 09, Revision E.01, M. J. Frisch, G. W. Trucks, H. B. Schlegel, G. E. Scuseria, M. A. Robb, J. R. Cheeseman, G. Scalmani, V. Barone, G. A. Petersson, H. Nakatsuji, X. Li, M. Caricato, A. Marenich, J. Bloino, B. G. Janesko, R. Gomperts, B. Mennucci, H. P. Hratchian, J. V. Ortiz, A. F. Izmaylov, J. L. Sonnenberg, D. Williams-Young, F. Ding, F. Lipparini, F. Egidi, J. Goings, B. Peng, A. Petrone, T. Henderson, D. Ranasinghe, V. G. Zakrzewski, J. Gao, N. Rega, G. Zheng, W. Liang, M. Hada, M. Ehara, K. Toyota, R. Fukuda, J. Hasegawa, M. Ishida, T. Nakajima, Y. Honda, O. Kitao, H. Nakai, T. Vreven, K. Throssell, J. A. Montgomery, Jr., J. E. Peralta, F. Ogliaro, M. Bearpark, J. J. Heyd, E. Brothers, K. N. Kudin, V. N. Staroverov, T. Keith, R. Kobayashi, J. Normand, K. Raghavachari, A. Rendell, J. C. Burant, S. S. Iyengar, J. Tomasi, M. Cossi, J. M. Millam, M. Klene, C. Adamo, R. Cammi, J. W. Ochterski, R. L. Martin, K. Morokuma, O. Farkas, J. B. Foresman, and D. J. Fox, Gaussian, Inc., Wallingford CT, 2016.

29. M. W. Schmidt, K. K. Baldridge, J. A. Boatz, S. T. Elbert, M. S. Gordon, J. H. Jensen, S. Koseki, N. Matsunaga, K. A. Nguyen, S. J. Su, T. L. Windus, M. Dupuis and J. A. Montgomery, Journal of computational chemistry, 1993, 14, 13471363.

30. J. B. Foresman, M. Headgordon, J. A. Pople and M. J. Frisch, J Phys Chem-Us, 1992, 96, 135-149.

31. C.-g. s. f. v. o. q. c. c. https://www.chemcraftprog.com.

32. D. E. Woon and T. H. Dunning, Journal of Chemical Physics, 1995, 103, 4572-4585.

33. H. HyperChem(TM) Professional 7.51, Inc., 1115 NW 4th Street, Gainesville, Florida 32601, USA.

34. V. Modi, S. Donnini, G. Groenhof and D. Morozov, The journal of physical chemistry. B, 2019, 123, $2325-2334$.

35. W. Humphrey, A. Dalke and K. Schulten, J Mol Graph Model, 1996, 14, 33-38.

36. A. D. MacKerell, D. Bashford, M. Bellott, R. L. Dunbrack, J. D. Evanseck, M. J. Field, S. Fischer, J. Gao, H. Guo, S. Ha, D. Joseph-McCarthy, L. Kuchnir, K. Kuczera, F. T. K. Lau, C. Mattos, S. Michnick, T. Ngo, D. T. Nguyen, B. Prodhom, W. E. Reiher, B. Roux, M. Schlenkrich, J. C. Smith, R. Stote, J. Straub, M. Watanabe, J. Wiorkiewicz-Kuczera, D. Yin and M. Karplus, Journal of Physical Chemistry B, 1998, 102, 3586-3616.

37. A. D. Mackerell, Journal of computational chemistry, 2004, 25, 1584-1604.

38. S. Kaminski, G. Daminelli and M. A. Mroginski, Journal of Physical Chemistry B, 2009, 113, 945-958.

39. S. Kaminski, 2010.

40. S. E. Feller, Y. H. Zhang, R. W. Pastor and B. R. Brooks, Journal of Chemical Physics, 1995, 103, 4613-4621.

41. G. J. Martyna, D. J. Tobias and M. L. Klein, Journal of Chemical Physics, 1994, 101, 4177-4189. 
